\title{
Existence Theory for Steady Flows of Fluids with Pressure and Shear Rate Dependent Viscosity, for Low Values of the Power-Law Index
}

\author{
M. Bulíček and V. Fišerová
}

\begin{abstract}
We deal with a system of partial differential equations describing a steady flow of a homogeneous incompressible non-Newtonian fluid with pressure and shear rate dependent viscosity subject to the homogeneous Dirichlet (no-slip) boundary condition. We establish a global existence of a weak solution for a certain class of such fluids in which the dependence of the viscosity on the shear rate is polynomiallike, characterized by the power-law index. A decomposition of the pressure and Lipschitz approximations of Sobolev functions are considered in order to obtain almost everywhere convergence of the pressure and the symmetric part of the velocity gradient and thus obtain new existence results for low value of the power-law index.
\end{abstract}

Keywords. Existence, weak solution, incompressible fluid, Lipschitz approximation of Sobolev functions, pressure- and shear-dependent material coefficients, decomposition of the pressure

Mathematics Subject Classification (2000). Primary 35Q30, secondary 76D03, $76 \mathrm{D} 05$

\section{Introduction}

In this paper we are interested in mathematical analysis of steady flows of an incompressible homogeneous non-Newtonian fluid in a bounded open domain $\Omega \subset \mathbb{R}^{d}(d \geq 2)$ that adheres to the boundary $\partial \Omega$. Such flows are described by the following governing equations

$$
\operatorname{div}(\boldsymbol{v} \otimes \boldsymbol{v})-\operatorname{div} \mathbf{T}=\boldsymbol{f} \text { in } \Omega, \quad \operatorname{div} \boldsymbol{v}=0 \quad \text { in } \Omega, \quad \boldsymbol{v}=\mathbf{0} \quad \text { on } \partial \Omega,
$$

M. Bulíček: Charles University in Prague, Faculty of Mathematics and Physics, Mathematical Institute, Sokolovská 83, 18675 Prague 8, Czech Republic;

mbul8060@karlin.mff.cuni.cz

V. Fišerová: Technische Universität Darmstadt, Fachbereich Mathematik, Schloßgartenstr. 7, 64289 Darmstadt, Germany; fiserova@mathematik.tu-darmstadt.de 
where $\boldsymbol{v}$ is the velocity of the fluid, $\boldsymbol{f}$ is the specific body force and $\mathbf{T}$ denotes the Cauchy stress tensor. We assume that the Cauchy stress takes the form

$$
\mathbf{T}=-p \mathbf{I}+\mathbf{S}(p, \mathbf{D}(\boldsymbol{v}))=-p \mathbf{I}+\nu\left(p,|\mathbf{D}(\boldsymbol{v})|^{2}\right) \mathbf{D}(\boldsymbol{v}),
$$

where $p$ is the pressure (normal mean stress), $\mathbf{S}$ is the constitutively determined part of the Cauchy stress and $\mathbf{D}(\boldsymbol{v})$ denotes the symmetric part of the velocity gradient. The dependence of the viscosity $\nu$ on $p$ and the second invariant of $\mathbf{D}(\boldsymbol{v})$ includes non-Newtonian features, as shear and pressure thinning and thickening, that the famous Navier-Stokes model is unable to capture.

Many experimental studies (see for example [5]) give clear evidence for the possible dependence of material coefficients on the pressure and models of the type (1.2) are often considered in various engineering areas, as in elastohydrodynamics or mechanics of granular and visco-elastic materials. It is worth noticing that there are at least two other concepts used in determining the form of the constitutive equation for $\mathbf{T}$ that provide a solid theoretical basis for models of the type (1.2). The key-words of the first approach, tabularly described in [27], are the maximization of the rate of dissipation w.r.t. the state variables and the structural constraints. The key-word of the second approach, originally formulated in [26], is the implicit constitutive theory. We refer to [21,22] where more details concerning the application of these concepts to (1.2) are addressed. The same sources $[21,22]$ provide also some explicit relationships between the Cauchy stress, the shear rate and the pressure and more related references.

In order to have a complete system of equations, we look for the pressure $p$ satisfying

$$
\int_{\Omega} p \mathrm{~d} x=p_{0} \equiv \text { const. }
$$

As it is not completely clear why we should assume such a "non-physical" condition, it requires a brief explanation. In the classical Navier-Stokes equations (or in the case when $\mathbf{S}(p, \mathbf{D}(\boldsymbol{v}))=\mathbf{S}(\mathbf{D}(\boldsymbol{v})$ ), i.e., the viscous stress tensor does not depend on the pressure) only the gradient of the pressure is met and so the choice of a constant that fixes the pressure is irrelevant. In our case, on the other hand, the situation is completely different. We deal with a pressure-dependent viscous stress and the choice of the constant $p_{0}$ may completely change the solution (not only the pressure $p$ but also the velocity $\boldsymbol{v}$ ). For details and some examples we refer to [15]. From the physical point of view it might be appropriate to prescribe the pressure locally, which leads in the context of weak solutions to prescribing the mean value of the pressure over some $\Omega_{0} \subset \Omega$. Note that the recent work [7] deals with exactly this condition. As such a generalization of (1.3) means essentially only technical difficulties, we do not consider it here. Moreover, we set $p_{0} \equiv 0$ for simplicity.

The arrangement of the paper is as follows. In the next section we introduce assumptions on $\mathbf{S}$ and also describe several important properties that the 
assumptions imply. We also fix the notation of the function spaces, formulate the main theorem of the paper and give some comments on previous results and new aspects of this paper. Then in Section 3 we recall several important lemmas that will be used in the proof. The $(\varepsilon, \eta)$-approximate problem is described in Section 4, where also several apriori estimates are derived. The last section is then devoted to the proof of the main theorem, i.e., to the limit procedures in $\varepsilon$ and $\eta$.

\section{Assumptions on $S$ and the main existence theorem}

2.1. Assumptions on $\mathbf{S}$. For arbitrarily fixed $r \in(1,2)$ we assume that $\mathbf{S}(p, \mathbf{D})$ introduced in (1.2) is a $\mathcal{C}^{1}$-mapping of $\mathbb{R} \times \mathbb{R}_{\mathrm{sym}}^{d \times d}$ into $\mathbb{R}_{\mathrm{sym}}^{d \times d}$ satisfying for all $\mathbf{D} \in \mathbb{R}_{\mathrm{sym}}^{d \times d}, \mathbf{B} \in \mathbb{R}_{\mathrm{sym}}^{d \times d}$ and $p \in \mathbb{R}$ the following two conditions:

$$
\begin{aligned}
C_{1}\left(1+|\mathbf{D}|^{2}\right)^{\frac{r-2}{2}}|\mathbf{B}|^{2} & \leq \frac{\partial \mathbf{S}_{i j}(p, \mathbf{D})}{\partial \mathbf{D}_{k l}} \mathbf{B}_{i j} \mathbf{B}_{k l} \leq C_{2}\left(1+|\mathbf{D}|^{2}\right)^{\frac{r-2}{2}}|\mathbf{B}|^{2} \\
\left|\frac{\partial \mathbf{S}(p, \mathbf{D})}{\partial p}\right| & \leq \gamma_{0}\left(1+|\mathbf{D}|^{2}\right)^{\frac{r-2}{4}} \leq \gamma_{0}<1,
\end{aligned}
$$

with some positive constants $C_{1}, C_{2}$ and $\gamma_{0}$. We refer to $[9,15]$ where several examples of $\mathbf{S}(p, \mathbf{D})$ that satisfy $(2.1)-(2.2)$ are considered.

Next, we recall several useful inequalities that are direct consequences of the assumptions (2.1)-(2.2). In order to simplify the notation, we define for arbitrary $\mathbf{A}, \mathbf{B} \in \mathbb{R}_{\mathrm{sym}}^{d \times d}$

$$
\mathcal{I}^{\mathbf{A}, \mathbf{B}}:=\int_{0}^{1}\left(1+|\mathbf{B}+s(\mathbf{A}-\mathbf{B})|^{2}\right)^{\frac{r-2}{2}}|\mathbf{A}-\mathbf{B}|^{2} \mathrm{~d} s .
$$

Then the monotonicity of $\mathbf{S}$ satisfying (2.1)-(2.2) is expressed by the following inequality:

$$
\frac{C_{1}}{2} \mathcal{I}^{\mathbf{A}, \mathbf{B}} \leq(\mathbf{S}(p, \mathbf{A})-\mathbf{S}(q, \mathbf{B})) \cdot(\mathbf{A}-\mathbf{B})+\frac{\gamma_{0}^{2}}{2 C_{1}}|p-q|^{2}
$$

that holds for arbitrary $p, q \in \mathbb{R}$. Another useful properties (as coercivity and growth conditions) follow from the condition (2.1), namely

$$
\mathbf{S}(p, \mathbf{D}) \cdot \mathbf{D} \geq \frac{C_{1}}{2 r}\left(|\mathbf{D}|^{r}-1\right)
$$

and

$$
|\mathbf{S}(p, \mathbf{D})| \leq \frac{C_{2}}{1-(2-r) \lambda}(1+|\mathbf{D}|)^{1-(2-r) \lambda} \quad \text { for all } \lambda \in[0,1] .
$$

For the proofs of (2.3), (2.4) and (2.5) see [9] and [23, Lemma 5.1.19], respectively. 
2.2. Main result. In what follows we consider that an open bounded domain $\Omega \subset \mathbb{R}^{d}, d \geq 2$, has Lipschitz boundary $\partial \Omega$ and we write $\Omega \in \mathcal{C}^{0,1}$. For $1 \leq q \leq \infty$ we define in the standard way the Lebesgue and Sobolev spaces $L^{q}(\Omega)$ and $W^{1, q}(\Omega)$ equipped with the norm $\|\cdot\|_{q}$ and $\|\cdot\|_{1, q}$, respectively. The Sobolev spaces of functions that vanish on the boundary $\partial \Omega$ are defined for $1 \leq q<\infty$ through $W_{0}^{1, q}(\Omega):=\overline{\mathcal{C}}_{0}^{\infty}(\Omega) \|_{1, q}$. We also use the following notation. If $X(\Omega)$ is a Banach space then $(X(\Omega))^{*}$ stands for its dual space and $X(\Omega)^{d}:=\left\{\boldsymbol{u}: \Omega \rightarrow \mathbb{R}^{d} ; u_{i} \in X(\Omega), i=1, \ldots, d\right\}$, similarly $X(\Omega)^{d \times d}:=\{\mathbf{A}:$ $\left.\Omega \rightarrow \mathbb{R}^{d \times d} ; \mathbf{A}_{i j} \in X(\Omega), i, j=1, \ldots, d\right\}$. Next, we introduce the subspaces of Lebesgue and Sobolev spaces

$$
\begin{aligned}
L_{0}^{q}(\Omega) & :=\left\{h \in L^{q}(\Omega): \int_{\Omega} h \mathrm{~d} x=0\right\} \\
W_{0, \operatorname{div}}^{1, q}(\Omega)^{d} & :=\left\{\boldsymbol{u} \in W_{0}^{1, q}(\Omega)^{d}: \operatorname{div} \boldsymbol{u}=0 \text { in } \Omega\right\} .
\end{aligned}
$$

Let us also denote the norm of the dual space $\left(W_{0}^{1, q}(\Omega)^{d}\right)^{*}=: W^{-1, q^{\prime}}(\Omega)^{d}$ by $\|\cdot\|_{-1, q^{\prime}}$ and the duality pairing by $\langle\cdot, \cdot\rangle, q^{\prime}=\frac{q}{q-1}$.

We are ready to formulate the result on the existence of a weak solution to (1.1)-(1.3) in the form of the following theorem.

Theorem 2.1 (Main theorem). Let $\Omega \in \mathcal{C}^{1}, d \geq 2$ and $\boldsymbol{f} \in W^{-1, r^{\prime}}(\Omega)^{d}$. Let $\frac{2 d}{d+2}<r<\min \left(2, \frac{3 d}{d+2}\right)$ and the assumptions (2.1) and (2.2) be satisfied with ${ }^{1}$

$$
\gamma_{0}<\frac{C_{1}}{C_{\text {div }}(\Omega, 2)\left(C_{1}+C_{2}\right)} .
$$

Then there exists a couple $(\boldsymbol{v}, p) \in W_{0, \mathrm{div}}^{1, r}(\Omega)^{d} \times L_{0}^{\frac{d r}{2(d-r)}}(\Omega)$ such that

$$
\begin{array}{r}
\int_{\Omega} \mathbf{S}(p, \mathbf{D}(\boldsymbol{v})) \cdot \mathbf{D}(\boldsymbol{\varphi}) \mathrm{d} x-\int_{\Omega}(\boldsymbol{v} \otimes \boldsymbol{v}) \cdot \nabla \boldsymbol{\varphi} \mathrm{d} x=\int_{\Omega} p \operatorname{div} \boldsymbol{\varphi} \mathrm{d} x+\langle\boldsymbol{f}, \boldsymbol{\varphi}\rangle \\
\text { for all } \boldsymbol{\varphi} \in \mathcal{C}_{0}^{\infty}(\Omega)^{d}
\end{array}
$$

The existence theory for steady flows of incompressible fluids with homogeneous Dirichlet boundary condition was first developed for the fluids with only shear rate dependent material coefficients. It was initiated in the late 1960s by Ladyzhenskaya (see [16-18]) and Lions (see [20]), showing the existence for $r \geq \frac{3 d}{d+2}$ with the use of standard arguments of the monotone operator theory. This result was later extended also for $r \geq \frac{2 d}{d+1}$ by Frehse, Málek and Steinhauer in [10] and by Růžička in [29]. The proof was based on the so-called $L^{\infty}$-truncation method, firstly introduced by Boccardo and Murat in [3], as well as on the monotone operator theory. Recently, Frehse, Málek and Steinhauer

\footnotetext{
${ }^{1}$ The constant $C_{\text {div }}(\Omega, 2)$ depends only on the shape of $\Omega$ and is introduced in Lemma 3.3.
} 
in [11] and Diening, Málek and Steinhauer in [8] established the existence of a weak solution for $r>\frac{2 d}{d+2}$ with the help of the Lipschitz approximations of Sobolev functions.

The existence of a weak solution for steady flows with $\mathbf{S}$ depending on the pressure and the shear rate subject to the homogeneous Dirichlet boundary condition was firstly established by Franta, Málek and Rajagopal in [9] for $\frac{3 d}{d+2}<r<2$. For the same range of parameters $r$ and for non-homogeneous Dirichlet boundary condition (but still for inner flows) we refer to [19]. For such cases it is enough to incorporate proper estimates on the pressure in the standard monotone operator theory because for $r \geq \frac{3 d}{d+2}$ the solution is a possible test function. Inspired by a result in $[8,11]$, we extend the theory presented in [9] for all $r>\frac{2 d}{d+2}$. For such values of the power-law index the Lipschitz approximation method is available.

However, in our case, i.e., $\frac{2 d}{d+2}<r \leq \frac{3 d}{d+2}$ and $\mathbf{S}$ depends on the pressure and the shear rate alike, the situation is even more tricky. For the reasons described in Section 4, we have to consider a more complicated approximate system with two levels of approximations and therefore to pass twice to the limit. The compactness of the velocity gradient and of the pressure has to be established in both limits, again with the help of the monotone operator condition (2.3). However, the application of (2.3) requires the pressure belonging to $L^{2}$, the assumption fulfilled in the case $\frac{3 d}{d+2}<r<2$, but not if $r \in\left(\frac{2 d}{d+2}, \frac{3 d}{d+2}\right)$. To overcome this problem, we decompose the pressure into two parts, one of them satisfying this assumption and the other one having some other properties.

Another new aspect of our study resides in an extension of the existence to an arbitrary dimension $d \geq 2$. While the condition $2>r>\frac{3 d}{d+2}$ implies that $d=2$ or 3 , for $\frac{3 d}{d+2}>r>\frac{2 d}{d+2}$ no such restriction is obtained because for all $d \geq 2$ we have $2>\frac{2 d}{d+2}$.

For the sake of completeness, we mention the known existence results for unsteady flows, where the Dirichlet boundary condition seems to present an interesting open problem. Málek, Nečas and Rajagopal in [24] and Hron, Málek, Nečas and Rajagopal in [15] showed global-in-time existence for incompressible fluids with pressure and shear rate dependent viscosity under spatially periodic boundary conditions, and these results were extended to flows in bounded domains subject to the Navier's slip by Bulíček, Málek and Rajagopal in [6]. Interestingly, for time dependent flows there are no results for a homogeneous Dirichlet boundary condition (that are in the context of time independent flows treated here).

On the other hand, there is no global existence theory available both for steady and unsteady flows of fluids whose viscosity depends only on the pressure. There are several studies, such as by Renardy [28], Gazzola [12] or Gazzola and Secchi [13], but all of them suffer from the drawback that either the structure 
of the viscosity is contradicted by experiments or only short-in-time existence of solutions for small data is shown. Recently, some analytical and numerical solutions for the flows of fluids with pressure-dependent viscosities in special geometries have been obtained by Hron, Málek and Rajagopal [14].

\section{Tools}

The purpose of this section is to introduce the necessary tools used later in the proof of the main theorem. The proof itself combines the standard approaches usually applied when dealing with mathematical analysis of problems similar to ours (as Galerkin method, compact embedding and Minty method) with more advanced methods such as the Lipschitz approximations of Sobolev functions, the $L^{p}$-solvability of the divergence equation and the $L^{q}$-theory for the steady Stokes system.

First, we recall the theory for the Stokes system.

Lemma 3.1 (Stokes operator, [2]). Let ${ }^{2} \Omega \in \mathcal{C}^{1}$ and $d \geq 2$. For any $1<q<\infty$, there exists a linear bounded operator $\mathcal{S}: W^{-1, q}(\Omega)^{d} \rightarrow W_{0, \mathrm{div}}^{1, q}(\Omega)^{d} \times L_{0}^{q}(\Omega)$ such that for $\boldsymbol{h} \in W^{-1, q}(\Omega)^{d}$ and $\mathcal{S}(\boldsymbol{h})=:\left(\mathcal{S}_{\boldsymbol{v}}(\boldsymbol{h}), \mathcal{S}_{p}(\boldsymbol{h})\right)$ the following equality holds in the sense of distributions:

$$
-\triangle \mathcal{S}_{\boldsymbol{v}}(\boldsymbol{h})+\nabla \mathcal{S}_{p}(\boldsymbol{h})=\boldsymbol{h} \quad \text { in } \Omega .
$$

The next crucial method used in the proof of the main theorem is the socalled Lipschitz approximation method. It is based on the fact that Sobolev functions from $W_{0}^{1, p}(\Omega), p \geq 1$, can be approximated by Lipschitz functions that differ from the original ones only on sets of small Lebesgue measures. Already Acerbi and Fusco in [1] showed their applications in the calculus of variations and since then they have been used by many others in various areas of analysis, for example in the existence theory of partial differential equations or in the regularity theory. We consider the Lipschitz approximations in the similar way as that in the study by Diening, Málek and Steinhauer [8], where also the following theorem that summarizes the important properties of the approximate functions together with more details and the proof can be found.

Lemma 3.2 (Lipschitz approximations, [8, Theorem 2.5]). Let $\Omega \in \mathcal{C}^{0,1}, d \geq 2$ and $1<p<\infty$. Let $\boldsymbol{u}^{n} \in W_{0}^{1, p}(\Omega)^{d}$ be such that $\boldsymbol{u}^{n} \rightarrow \mathbf{0}$ weakly in $W_{0}^{1, p}(\bar{\Omega})^{d}$ as $n \rightarrow \infty$. We set

$$
\begin{aligned}
K & :=\sup _{n}\left\|\boldsymbol{u}^{n}\right\|_{1, p}<\infty \\
\gamma_{n} & :=\left\|\boldsymbol{u}^{n}\right\|_{p} \rightarrow 0 \quad(n \rightarrow \infty) .
\end{aligned}
$$

\footnotetext{
${ }^{2}$ Lemma 3.1 holds also for $\Omega \in \mathcal{C}^{0}, 1$ for $d=3$ and for $q \in\left(\frac{3}{2}, 3\right)$. Since we need also $q \leq \frac{3}{2}$ in the paper, we do not formulate the Lemma in such generality.
} 
Let $\theta_{n}>0$ be such that $\left(\right.$ e.g. $\left.\theta_{n}:=\sqrt{\gamma_{n}}\right) \theta_{n} \rightarrow 0$ and $\frac{\gamma_{n}}{\theta_{n}} \rightarrow 0(n \rightarrow \infty)$. Let $\mu_{j}:=2^{2^{j}}$. Then there exist a sequence $\lambda_{n, j}$ with

$$
\mu_{j} \leq \lambda_{n, j} \leq \mu_{j+1}
$$

and a sequence $\boldsymbol{u}^{n, j} \in W_{0}^{1, \infty}(\Omega)^{d}$ such that for all $j, n \in \mathbb{N}$

$$
\begin{aligned}
\left\|\boldsymbol{u}^{n, j}\right\|_{\infty} & \leq \theta_{n} \rightarrow 0 \quad(n \rightarrow \infty) \\
\left\|\nabla \boldsymbol{u}^{n, j}\right\|_{\infty} & \leq c \lambda_{n, j} \leq c \mu_{j+1}
\end{aligned}
$$

and $\left(\mathcal{M}\right.$ denotes the Hardy-Littlewood maximal function $\left.{ }^{3}\right)$

$$
\left\{\boldsymbol{u}^{n} \neq \boldsymbol{u}^{n, j}\right\} \subset \Omega \cap\left(\left\{\mathcal{M}\left(\boldsymbol{u}^{n}\right)>\theta_{n}\right\} \cup\left\{\mathcal{M}\left(\nabla \boldsymbol{u}^{n}\right)>2 \lambda_{n, j}\right\}\right)
$$

Moreover, for all $j \in \mathbb{N}$ and $n \rightarrow \infty$

$$
\begin{array}{rlr}
\boldsymbol{u}^{n, j} \rightarrow \mathbf{0} & \text { strongly in } L^{q}(\Omega)^{d} & \forall q \in[1, \infty] \\
\boldsymbol{u}^{n, j} \rightarrow \mathbf{0} & \text { weakly in } W_{0}^{1, q}(\Omega)^{d} & \forall q \in[1, \infty) \\
\nabla \boldsymbol{u}^{n, j} \stackrel{*}{\rightarrow} \mathbf{0} & \text { weakly }^{*} \text { in } L^{\infty}(\Omega)^{d \times d} &
\end{array}
$$

and for all $n, j \in \mathbb{N}$

$$
\left\|\nabla \boldsymbol{u}^{n, j} \chi_{\left\{\boldsymbol{u}^{n} \neq \boldsymbol{u}^{n, j}\right\}}\right\|_{p} \leq c\left\|\lambda_{n, j} \chi_{\left\{\boldsymbol{u}^{n} \neq \boldsymbol{u}^{n, j}\right\}}\right\|_{p} \leq c \frac{\gamma_{n}}{\theta_{n}} \mu_{j+1}+c \varepsilon_{j},
$$

where $\varepsilon_{j}:=K 2^{-\frac{j}{p}}$ vanishes as $j \rightarrow \infty$ and the constant $c$ depends on $\Omega$.

The last important tool that we will often use in the proof is the $L^{p_{-}}$ solvability of the Dirichlet problem for the divergence equation (firstly addressed in $[4])$.

Lemma 3.3 (Bogovskiü operator, [25, Lemma 3.17]). Let $\Omega \in \mathcal{C}^{0,1}$ and $d \geq 2$. Then there exists a continuous linear operator $\mathcal{B}: L_{0}^{p}(\Omega) \rightarrow W_{0}^{1, p}(\Omega)^{d}$ such that for all $1<p<\infty$ and for all $f \in L_{0}^{p}(\Omega)$

$$
\operatorname{div}(\mathcal{B} f)=f \quad \text { in } \Omega, \quad\|\mathcal{B} f\|_{1, p} \leq C_{\operatorname{div}}(\Omega, p)\|f\|_{p}
$$

Moreover, if $f=\operatorname{div} \boldsymbol{u}$ and $\boldsymbol{u} \in W_{0}^{1, p}(\Omega)^{d} \cap L^{q}(\Omega)^{d}$ with some $1<q<\infty$, then there exists a constant $C(\Omega, q)$ such that $\|\mathcal{B} f\|_{q} \leq C(\Omega, q)\|\boldsymbol{u}\|_{q}$.

\footnotetext{
${ }^{3}$ For a function $f \in L^{1}\left(\mathbb{R}^{d}\right)$, we define the Hardy-Littlewood maximal function through $(\mathcal{M} f)(x):=\sup _{r>0} \frac{1}{\left|B_{r}(x)\right|} \int_{B_{r}(x)}|f(y)| \mathrm{d} y$.
} 


\section{Approximate system and its properties}

In order to establish the existence of a weak solution to (1.1)-(1.3), we first introduce the $(\varepsilon, \eta)$-approximate system. The $\varepsilon$-approximation is considered since we need to control the pressure from the very beginning. Therefore, we replace the constraint of incompressibility $\operatorname{div} \boldsymbol{v}=0$ by the following Neumann problem for the pressure of the form $(\varepsilon>0)$

$$
-\varepsilon \triangle p^{\varepsilon}+\varepsilon p^{\varepsilon}+\operatorname{div} \boldsymbol{v}^{\varepsilon}=0 \quad \text { in } \Omega, \quad \frac{\partial p^{\varepsilon}}{\partial \boldsymbol{n}}=0 \quad \text { on } \partial \Omega .
$$

In order to be able to test with the solution itself, we consider $\eta$-approximation by introducing an extra term to the equation of linear momentum $(1.1)_{1}$, namely we add the term $\eta\left|\boldsymbol{v}^{\varepsilon, \eta}\right|^{2 r^{\prime}-2} \boldsymbol{v}^{\varepsilon, \eta}$, where $r^{\prime}=\frac{r}{r-1}$. Since $\operatorname{div} \boldsymbol{v}^{\varepsilon, \eta}$ is no longer equal to zero and we would still like to deal easily with the convective term (for preservation of uniform estimates), we modify it as well. For this purpose, we define the "Bogovskiu " projection $\mathcal{P}: W_{0}^{1, q}(\Omega)^{d} \rightarrow W_{0, \mathrm{div}}^{1, q}(\Omega)^{d}$ as

$$
\mathcal{P} \boldsymbol{v}:=\boldsymbol{v}-\mathcal{B}(\operatorname{div} \boldsymbol{v})
$$

Note that from the definition it is obvious that $\operatorname{div} \mathcal{P} \boldsymbol{v}=0$ a.e. in $\Omega$ and therefore considering $\boldsymbol{v}^{\varepsilon, \eta} \otimes \mathcal{P} \boldsymbol{v}^{\varepsilon, \eta}$ instead of $\boldsymbol{v}^{\varepsilon, \eta} \otimes \boldsymbol{v}^{\varepsilon, \eta}$ gives

$$
\int_{\Omega}\left(\boldsymbol{v}^{\varepsilon, \eta} \otimes \mathcal{P} \boldsymbol{v}^{\varepsilon, \eta}\right) \cdot \nabla \boldsymbol{v}^{\varepsilon, \eta} \mathrm{d} x=0
$$

Later on, we will see that thanks to the additional term $\eta\left|\boldsymbol{v}^{\varepsilon, \eta}\right|^{2 r^{\prime}-2} \boldsymbol{v}^{\varepsilon, \eta}$ the expression $\left(\boldsymbol{v}^{\varepsilon, \eta} \otimes \mathcal{P} \boldsymbol{v}^{\varepsilon, \eta}\right) \cdot \nabla \boldsymbol{v}^{\varepsilon, \eta}$ is indeed an integrable function. Moreover, from the properties of the Bogovskil operator (Lemma 3.3) we also have the following estimates

$$
\left\|\mathcal{P} \boldsymbol{v}^{\varepsilon, \eta}\right\|_{1, r} \leq C\left\|\boldsymbol{v}^{\varepsilon, \eta}\right\|_{1, r}, \quad\left\|\mathcal{P} \boldsymbol{v}^{\varepsilon, \eta}\right\|_{2 r^{\prime}} \leq C\left\|\boldsymbol{v}^{\varepsilon, \eta}\right\|_{2 r^{\prime}}
$$

Incorporating all of the above-mentioned modifications, we obtain the $(\varepsilon, \eta)$ approximate system of the following form:

$$
\begin{aligned}
\eta\left|\boldsymbol{v}^{\varepsilon, \eta}\right|^{2 r^{\prime}-2} \boldsymbol{v}^{\varepsilon, \eta}+\operatorname{div}\left(\boldsymbol{v}^{\varepsilon, \eta} \otimes \mathcal{P} \boldsymbol{v}^{\varepsilon, \eta}\right)-\operatorname{div}\left(\mathbf{S}\left(p^{\varepsilon, \eta}, \mathbf{D}\left(\boldsymbol{v}^{\varepsilon, \eta}\right)\right)+\nabla p^{\varepsilon, \eta}\right. & =\boldsymbol{f} \\
-\varepsilon \triangle p^{\varepsilon, \eta}+\varepsilon p^{\varepsilon, \eta}+\operatorname{div} \boldsymbol{v}^{\varepsilon, \eta} & =0,
\end{aligned}
$$

completed by the boundary conditions $\boldsymbol{v}^{\varepsilon, \eta}=\mathbf{0}$ and $\frac{\partial p^{\varepsilon, \eta}}{\partial \boldsymbol{n}}=0$ on $\partial \Omega$. Note that (4.3) implies that $\int_{\Omega} p^{\varepsilon, \eta} \mathrm{d} x=0$.

The following lemma establishes the existence of a weak solution to $(\varepsilon, \eta)$ approximative system and shows the uniform estimates fulfilled by the approximate velocity $\boldsymbol{v}^{\varepsilon, \eta}$ and the approximate pressure $p^{\varepsilon, \eta}$. 
Lemma 4.1. Let $\varepsilon, \eta>0$ be arbitrary. Let all assumptions of Theorem 2.1 be satisfied. Then there exists a couple $\left(\boldsymbol{v}^{\varepsilon, \eta}, p^{\varepsilon, \eta}\right)$ satisfying

$$
\begin{gathered}
\boldsymbol{v}^{\varepsilon, \eta} \in W_{0}^{1, r}(\Omega)^{d} \cap L^{2 r^{\prime}}(\Omega)^{d} \quad \text { and } \quad p^{\varepsilon, \eta} \in W^{1,2}(\Omega) \cap L_{0}^{2}(\Omega), \\
\varepsilon \int_{\Omega} \nabla p^{\varepsilon, \eta} \cdot \nabla \xi \mathrm{d} x+\varepsilon \int_{\Omega} p^{\varepsilon, \eta} \xi \mathrm{d} x+\int_{\Omega} \operatorname{div} \boldsymbol{v}^{\varepsilon, \eta} \xi \mathrm{d} x=0 \\
\text { for all } \xi \in W^{1,2}(\Omega),
\end{gathered}
$$

and

$$
\begin{array}{r}
\eta \int_{\Omega}\left|\boldsymbol{v}^{\varepsilon, \eta}\right|^{2 r^{\prime}-2} \boldsymbol{v}^{\varepsilon, \eta} \cdot \boldsymbol{\varphi} \mathrm{d} x+\int_{\Omega} \mathbf{S}\left(p^{\varepsilon, \eta}, \mathbf{D}\left(\boldsymbol{v}^{\varepsilon, \eta}\right)\right) \cdot \mathbf{D}(\boldsymbol{\varphi}) \mathrm{d} x \\
-\int_{\Omega}\left(\boldsymbol{v}^{\varepsilon, \eta} \otimes \mathcal{P} \boldsymbol{v}^{\varepsilon, \eta}\right) \cdot \nabla \boldsymbol{\varphi} \mathrm{d} x-\int_{\Omega} p^{\varepsilon, \eta} \operatorname{div} \boldsymbol{\varphi} \mathrm{d} x=\langle\boldsymbol{f}, \boldsymbol{\varphi}\rangle \\
{\left[0.1 \text { cm] for all } \boldsymbol{\varphi} \in W_{0}^{1, r}(\Omega)^{d} \cap L^{2 r^{\prime}}(\Omega)^{d} .\right.}
\end{array}
$$

Moreover,

$$
\varepsilon\left\|\nabla p^{\varepsilon, \eta}\right\|_{2}^{2}+\varepsilon\left\|p^{\varepsilon, \eta}\right\|_{2}^{2}+C\left\|\nabla \boldsymbol{v}^{\varepsilon, \eta}\right\|_{r}^{r}+\eta\left\|\boldsymbol{v}^{\varepsilon, \eta}\right\|_{2 r^{\prime}}^{2 r^{\prime}} \leq C<\infty
$$

and consequently,

$$
\left\|\mathbf{S}\left(p^{\varepsilon, \eta}, \mathbf{D}\left(\boldsymbol{v}^{\varepsilon, \eta}\right)\right)\right\|_{r^{\prime}} \leq C<\infty .
$$

Furthermore, the pressure satisfies the following uniform estimate with respect to $\varepsilon$ :

$$
\left\|p^{\varepsilon, \eta}\right\|_{\frac{2 d r}{(d-2) r+d}} \leq C(\eta)<\infty .
$$

Proof. First of all, note that all the integrals above make sense, including the integral $\int_{\Omega} \operatorname{div} \boldsymbol{v}^{\varepsilon, \eta} \xi \mathrm{d} x$ as $W^{1,2}(\Omega) \hookrightarrow L^{r^{\prime}}(\Omega)$ for $r>\frac{2 d}{d+2}$. The existence of $\left(\boldsymbol{v}^{\varepsilon, \eta}, p^{\varepsilon, \eta}\right)$ fulfilling (4.4)-(4.6) can be proven via Galerkin approximations with the help of the compact embedding and the monotonicity condition (2.3). Brouwer's fixed point theorem, properties (4.1) of the projection $\mathcal{P} \boldsymbol{v}^{\varepsilon, \eta}$ and Vitali's theorem has to be used. The proof then follows the same pattern as in [9]. We comment the derivation of inequalities (4.7)-(4.9) only.

Taking $\xi:=p^{\varepsilon, \eta}$ in (4.5) and $\boldsymbol{\varphi}:=\boldsymbol{v}^{\varepsilon, \eta}$ in (4.6), adding resulting equations and using Korn's, Young's and Poincaré's inequalities lead to the estimate (4.7). Inequality (4.8) then follows from the growth condition (2.5) with $\lambda=1$.

In order to obtain the estimate (4.9) on the pressure $p^{\varepsilon, \eta}$, we set $\boldsymbol{\varphi}=\boldsymbol{\varphi}^{\varepsilon, \eta}:=$ $\mathcal{B}\left(\left|p^{\varepsilon, \eta}\right|^{s-2} p^{\varepsilon, \eta}-\frac{1}{|\Omega|} \int_{\Omega}\left|p^{\varepsilon, \eta}\right|^{s-2} p^{\varepsilon, \eta} \mathrm{d} x\right)$ in (4.6) with $s=\frac{2 d r}{(d-2) r+d}$. Note that from the properties of the Bogovskil operator $\varphi^{\varepsilon, \eta}$ satisfies

$$
\left\|\boldsymbol{\varphi}^{\varepsilon, \eta}\right\|_{1, s^{\prime}} \leq 2 C_{\operatorname{div}}\left(\Omega, s^{\prime}\right)\left\|p^{\varepsilon, \eta}\right\|_{s}^{s-1} \text {. }
$$


We use the fact that $\int_{\Omega} p^{\varepsilon, \eta} \mathrm{d} x=0$ and with the help of (2.5), (4.7), (4.10) and Sobolev embeddings, namely $W^{1, s^{\prime}}(\Omega) \hookrightarrow L^{2 r^{\prime}}(\Omega)$, we can conclude (note that $r \leq s^{\prime}$ and $\left.s \leq r^{\prime}\right)$

$$
\begin{aligned}
\left\|p^{\varepsilon, \eta}\right\|_{s}^{s}= & \int_{\Omega} \mathbf{S}\left(p^{\varepsilon, \eta}, \mathbf{D}\left(\boldsymbol{v}^{\varepsilon, \eta}\right)\right) \cdot \mathbf{D}\left(\boldsymbol{\varphi}^{\varepsilon, \eta}\right) \mathrm{d} x-\left\langle\boldsymbol{f}, \boldsymbol{\varphi}^{\varepsilon, \eta}\right\rangle \\
& -\int_{\Omega}\left(\boldsymbol{v}^{\varepsilon, \eta} \otimes \mathcal{P} \boldsymbol{v}^{\varepsilon, \eta}\right) \cdot \nabla \boldsymbol{\varphi}^{\varepsilon, \eta} \mathrm{d} x+\eta \int_{\Omega}\left|\boldsymbol{v}^{\varepsilon, \eta}\right|^{2 r^{\prime}-2} \boldsymbol{v}^{\varepsilon, \eta} \cdot \boldsymbol{\varphi}^{\varepsilon, \eta} \mathrm{d} x \\
\leq & C\left(1+\left\|\mathbf{D}\left(\boldsymbol{v}^{\varepsilon, \eta}\right)\right\|_{r}\right)^{r-1}\left\|\boldsymbol{\varphi}^{\varepsilon, \eta}\right\|_{1, r}+\|\boldsymbol{f}\|_{-1, r^{\prime}}\left\|\boldsymbol{\varphi}^{\varepsilon, \eta}\right\|_{1, r} \\
& +\left\|\boldsymbol{v}^{\varepsilon, \eta} \otimes \mathcal{P} \boldsymbol{v}^{\varepsilon, \eta}\right\|_{s}\left\|\nabla \boldsymbol{\varphi}^{\varepsilon, \eta}\right\|_{s^{\prime}}+\eta\left\|\boldsymbol{v}^{\varepsilon, \eta}\right\|_{2 r^{\prime}}^{2 r^{\prime}-1}\left\|\boldsymbol{\varphi}^{\varepsilon, \eta}\right\|_{2 r^{\prime}} \\
\leq & C(\eta)\left\|\boldsymbol{\varphi}^{\varepsilon, \eta}\right\|_{1, s^{\prime}} \leq C(\eta)\left\|p^{\varepsilon, \eta}\right\|_{s}^{s-1},
\end{aligned}
$$

which leads to the desired estimate (4.9).

\section{Proof of the main theorem}

This final section is devoted to the proof of Theorem 2.1. The proof is split into several steps that will be discussed in the following subsections. In order to obtain a weak solution to (1.1)-(1.3), we first recall $(\varepsilon, \eta)$-approximate system introduced in the previous section and let first $\varepsilon$ and then $\eta$ tend to 0 . In both limits the difficulty occurs in the viscous stress tensor because it depends on the pressure and on the shear rate nonlinearly. Therefore, several extra tools are needed such as a decomposition of the pressure or the Lipschitz approximations of Sobolev functions.

5.1. Limit $\varepsilon \rightarrow 0$. For simplicity, we denote $\left(\boldsymbol{v}^{\varepsilon}, p^{\varepsilon}\right):=\left(\boldsymbol{v}^{\varepsilon, \eta}, p^{\varepsilon, \eta}\right)$ in this subsection, where $\left(\boldsymbol{v}^{\varepsilon, \eta}, p^{\varepsilon, \eta}\right)$ is the couple introduced in Lemma 4.1. First of all, the estimates (4.7), (4.8) and (4.9) allow us to find a (not relabeled) subsequence $\left(\boldsymbol{v}^{\varepsilon}, p^{\varepsilon}\right)$ and a couple $(\boldsymbol{v}, p) \in\left(W_{0}^{1, r}(\Omega)^{d} \cap L^{2 r^{\prime}}(\Omega)^{d}\right) \times L_{0}^{\frac{2 d r}{(d-2) r+d}}(\Omega)$ such that

$$
\begin{aligned}
\boldsymbol{v}^{\varepsilon} \rightarrow \boldsymbol{v} & \text { weakly in } W_{0}^{1, r}(\Omega)^{d} \\
\boldsymbol{v}^{\varepsilon} \rightarrow \boldsymbol{v} & \text { weakly in } L^{2 r^{\prime}}(\Omega)^{d} \\
p^{\varepsilon} \rightarrow p & \text { weakly in } L_{0}^{\frac{2 d r}{(d-2) r+d}}(\Omega) \\
\mathbf{S}\left(p^{\varepsilon}, \mathbf{D}\left(\boldsymbol{v}^{\varepsilon}\right)\right) \rightarrow \overline{\mathbf{S}} & \text { weakly in } L^{r^{\prime}}(\Omega)^{d \times d},
\end{aligned}
$$

and due to the compact embedding and the interpolation inequality

$$
\begin{aligned}
\boldsymbol{v}^{\varepsilon} & \rightarrow \boldsymbol{v} & & \text { strongly in } L^{q}(\Omega)^{d} \text { for all } 1 \leq q<\frac{d r}{d-r} \\
\boldsymbol{v}^{\varepsilon} & \rightarrow \boldsymbol{v} & & \text { strongly in } L^{q}(\Omega)^{d} \text { for all } 1 \leq q<2 r^{\prime} \\
\left|\boldsymbol{v}^{\varepsilon}\right|^{2 r^{\prime}-2} \boldsymbol{v}^{\varepsilon} & \rightarrow|\boldsymbol{v}|^{2 r^{\prime}-2} \boldsymbol{v} & & \text { weakly in } L^{\frac{2 r^{\prime}}{2 r^{\prime}-1}}(\Omega)^{d} .
\end{aligned}
$$


We would like to pass to the limit in the identities (4.5) and (4.6). Doing so, it follows directly from the first identity and from (4.7) that

$$
\operatorname{div} \boldsymbol{v}=0 \quad \text { a.e. in } \Omega \text {. }
$$

This fact helps us to treat the convective term. Indeed, (5.2) 2 and (5.3) imply that

$$
\mathcal{P} \boldsymbol{v}^{\varepsilon} \rightarrow \boldsymbol{v} \text { strongly in } L^{q}(\Omega)^{d} \text { for all } q: 1 \leq q<2 r^{\prime},
$$

which can be gained from the definition of $\mathcal{P} \boldsymbol{v}^{\varepsilon}$ and from the linearity and continuity of the Bogovskil operator $\mathcal{B}$ (Lemma 3.3). Therefore, using (5.1)(5.4), one can easily deduce that

$$
\begin{aligned}
& \eta \int_{\Omega}|\boldsymbol{v}|^{2 r^{\prime}-2} \boldsymbol{v} \cdot \boldsymbol{\varphi} \mathrm{d} x-\int_{\Omega}(\boldsymbol{v} \otimes \boldsymbol{v}) \cdot \nabla \boldsymbol{\varphi} \mathrm{d} x+\int_{\Omega} \overline{\mathbf{S}} \cdot \mathbf{D}(\boldsymbol{\varphi}) \mathrm{d} x \\
& =\int_{\Omega} p \operatorname{div} \boldsymbol{\varphi} \mathrm{d} x+\langle\boldsymbol{f}, \boldsymbol{\varphi}\rangle \quad \text { for all } \boldsymbol{\varphi} \in W^{1, \infty}(\Omega)^{d} \cap W_{0}^{1, r}(\Omega)^{d} .
\end{aligned}
$$

It remains to identify $\overline{\mathbf{S}}=\mathbf{S}(p, \mathbf{D}(\boldsymbol{v}))$ a.e. in $\Omega$. To show this, it is enough to prove that

$$
p^{\varepsilon} \rightarrow p \quad \text { a.e. in } \Omega \quad \text { and } \quad \mathbf{D}\left(\boldsymbol{v}^{\varepsilon}\right) \rightarrow \mathbf{D}(\boldsymbol{v}) \text { a.e. in } \Omega
$$

at least for a subsequence. Vitali's theorem then completes this part of the proof.

In order to show (5.6), we first decompose the pressure $p^{\varepsilon}$ into two particular pressures. The first one will converge strongly in some Lebesgue space and the second one only weakly but in some "better" Lebesgue space, namely in $L^{r^{\prime}}(\Omega)$. As a second step, we recall the monotonicity condition (2.3) for $\mathbf{S}$ and with the help it we will be able to prove (5.6).

For the decomposition of the pressure, we consider two Stokes problems (3.1) with the right-hand sides $\boldsymbol{h}_{i}=\boldsymbol{h}_{i}^{\varepsilon}(i=1,2)$ of the form

$$
\begin{array}{ll}
\boldsymbol{h}_{1}^{\varepsilon}=\operatorname{div}\left(\mathbf{S}\left(p^{\varepsilon}, \mathbf{D}\left(\boldsymbol{v}^{\varepsilon}\right)\right)\right)+\boldsymbol{f} & \in\left(W_{0}^{1, r}(\Omega)\right)^{*} \\
\boldsymbol{h}_{2}^{\varepsilon}=-\operatorname{div}\left(\boldsymbol{v}^{\varepsilon} \otimes \mathcal{P} \boldsymbol{v}^{\varepsilon}\right)-\eta\left|\boldsymbol{v}^{\varepsilon}\right|^{2 r^{\prime}-2} \boldsymbol{v}^{\varepsilon} \in\left(W_{0}^{1, s^{\prime}}(\Omega)\right)^{*}, \quad s^{\prime}:=\frac{2 d r}{(d+2) r-d}
\end{array}
$$

and we denote $\left(\boldsymbol{v}_{i}^{\varepsilon}, p_{i}^{\varepsilon}\right):=\mathcal{S}\left(\boldsymbol{h}_{i}^{\varepsilon}\right), i=1,2$. Lemma 3.1 then implies

$$
\begin{aligned}
& \left\|\nabla \boldsymbol{v}_{1}^{\varepsilon}\right\|_{r^{\prime}}+\left\|p_{1}^{\varepsilon}\right\|_{r^{\prime}} \leq C\left\|\boldsymbol{h}_{1}^{\varepsilon}\right\|_{\left(W_{0}^{1, r}(\Omega)\right)^{*}} \leq C+C\left\|\mathbf{S}\left(p^{\varepsilon}, \mathbf{D}\left(\boldsymbol{v}^{\varepsilon}\right)\right)\right\|_{r^{\prime}} \stackrel{(4.8)}{\leq} C \\
& \left\|\nabla \boldsymbol{v}_{2}^{\varepsilon}\right\|_{s}+\left\|p_{2}^{\varepsilon}\right\|_{s} \leq C\left\|\boldsymbol{h}_{2}^{\varepsilon}\right\|_{\left(W_{0}^{1, s^{\prime}}(\Omega)\right)^{*}} \leq C\left\|\boldsymbol{v}^{\varepsilon} \otimes \mathcal{P} \boldsymbol{v}^{\varepsilon}\right\|_{s}+C \eta\left\|\boldsymbol{v}^{\varepsilon}\right\|_{2 r^{\prime}}^{2 r^{\prime}-1} \stackrel{(4.7)}{\leq} C\left(\eta^{-1}\right) .
\end{aligned}
$$


Owing to the strong convergence result $(5.2)_{2}$ and the linearity of $\mathcal{S}$, we get

$$
\begin{array}{cl}
\nabla \boldsymbol{v}_{1}^{\varepsilon} \rightarrow \nabla \boldsymbol{v}_{1} & \text { weakly in } L^{r^{\prime}}(\Omega)^{d \times d} \\
p_{1}^{\varepsilon} \rightarrow p_{1} & \text { weakly in } L^{r^{\prime}}(\Omega) \\
\nabla \boldsymbol{v}_{2}^{\varepsilon} \rightarrow \nabla \boldsymbol{v}_{2} & \text { strongly in } L^{q}(\Omega)^{d \times d} \\
p_{2}^{\varepsilon} \rightarrow p_{2} & \text { strongly in } L^{q}(\Omega),
\end{array}
$$

where $q \in\left[1, \frac{2 d r}{(d-2) r+d}\right)$. Using (4.6) and the linearity of $\mathcal{S}$ we deduce that $\mathcal{S}\left(\boldsymbol{h}_{1}\right)+\mathcal{S}\left(\boldsymbol{h}_{2}\right)=\mathcal{S}\left(\boldsymbol{h}_{1}+\boldsymbol{h}_{2}\right)=\mathcal{S}\left(\nabla p^{\varepsilon}\right)=\left(\mathbf{0}, \nabla p^{\varepsilon}\right)$. Consequently,

$$
p^{\varepsilon}=p_{1}^{\varepsilon}+p_{2}^{\varepsilon} \quad \text { and } \quad \boldsymbol{v}_{1}^{\varepsilon}=-\boldsymbol{v}_{2}^{\varepsilon},
$$

and by using $(5.7)_{3}$ we obtain

$$
\nabla \boldsymbol{v}_{1}^{\varepsilon} \rightarrow \nabla \boldsymbol{v}_{1} \quad \text { strongly in } L^{q}(\Omega)^{d \times d}
$$

Next, setting $\mathbf{A}:=\mathbf{D}\left(\boldsymbol{v}^{\varepsilon}\right), \mathbf{B}:=\mathbf{D}(\boldsymbol{v}), p:=p^{\varepsilon}$ and $q:=p_{1}+p_{2}^{\varepsilon}$ in (2.3) and integrating it over the domain $\Omega$, we have

$$
\begin{aligned}
& \frac{C_{1}}{2} \int_{\Omega} \mathcal{I}^{\mathbf{D}\left(\boldsymbol{v}^{\varepsilon}\right), \mathbf{D}(\boldsymbol{v})} \mathrm{d} x \\
& \leq \int_{\Omega}\left(\mathbf{S}\left(p^{\varepsilon}, \mathbf{D}\left(\boldsymbol{v}^{\varepsilon}\right)\right)-\mathbf{S}\left(p_{1}+p_{2}^{\varepsilon}, \mathbf{D}(\boldsymbol{v})\right)\right) \cdot\left(\mathbf{D}\left(\boldsymbol{v}^{\varepsilon}\right)-\mathbf{D}(\boldsymbol{v})\right) \mathrm{d} x \\
& \quad+\frac{\gamma_{0}^{2}}{2 C_{1}}\left\|p_{1}^{\varepsilon}-p_{1}\right\|_{2}^{2} .
\end{aligned}
$$

Our goal is to show that $\int_{\Omega} \mathcal{I}^{\mathbf{D}\left(\boldsymbol{v}^{\varepsilon}\right), \mathbf{D}(\boldsymbol{v})} \mathrm{d} x \rightarrow 0$ for $\varepsilon \rightarrow 0$.

First, since $p_{2}^{\varepsilon}$ converges (for a not relabeled sequence) a.e. in $\Omega,(2.5)$ with $\lambda=1$ and Lebesgue's dominated convergence theorem imply that

$$
\mathbf{S}\left(p_{1}+p_{2}^{\varepsilon}, \mathbf{D}(\boldsymbol{v})\right) \rightarrow \mathbf{S}(p, \mathbf{D}(\boldsymbol{v})) \quad \text { strongly in } L^{r^{\prime}}(\Omega)^{d \times d} .
$$

Therefore,

$$
\int_{\Omega} \mathbf{S}\left(p_{1}+p_{2}^{\varepsilon}, \mathbf{D}(\boldsymbol{v})\right) \cdot\left(\mathbf{D}\left(\boldsymbol{v}^{\varepsilon}\right)-\mathbf{D}(\boldsymbol{v})\right) \mathrm{d} x \rightarrow 0 \quad \text { for } \varepsilon \rightarrow 0 .
$$

Next, considering the weak formulation (4.6) with $\boldsymbol{\varphi}:=\boldsymbol{v}^{\varepsilon}-\boldsymbol{v}$, we arrive at

$$
\begin{aligned}
\int_{\Omega} \mathbf{S}\left(p^{\varepsilon}, \mathbf{D}\left(\boldsymbol{v}^{\varepsilon}\right)\right) \cdot \mathbf{D}\left(\boldsymbol{v}^{\varepsilon}-\boldsymbol{v}\right) \mathrm{d} x= & \int_{\Omega} p^{\varepsilon} \operatorname{div}\left(\boldsymbol{v}^{\varepsilon}-\boldsymbol{v}\right) \mathrm{d} x+\left\langle\boldsymbol{f}, \boldsymbol{v}^{\varepsilon}-\boldsymbol{v}\right\rangle \\
& +\int_{\Omega}\left(\boldsymbol{v}^{\varepsilon} \otimes \mathcal{P} \boldsymbol{v}^{\varepsilon}\right) \cdot \nabla\left(\boldsymbol{v}^{\varepsilon}-\boldsymbol{v}\right) \mathrm{d} x \\
& -\eta \int_{\Omega}\left|\boldsymbol{v}^{\varepsilon}\right|^{2 r^{\prime}-2} \boldsymbol{v}^{\varepsilon} \cdot\left(\boldsymbol{v}^{\varepsilon}-\boldsymbol{v}\right) \mathrm{d} x
\end{aligned}
$$


As $\operatorname{div} \boldsymbol{v}=0$ a.e. in $\Omega$ and $\int_{\Omega} p^{\varepsilon} \operatorname{div} \boldsymbol{v}^{\varepsilon} \mathrm{d} x=-\varepsilon\left\|\nabla p^{\varepsilon}\right\|_{2}^{2}-\varepsilon\left\|p^{\varepsilon}\right\|_{2}^{2}$, which follows from (4.5) with $\xi:=p^{\varepsilon}$, and since the terms $\varepsilon\left\|\nabla p^{\varepsilon}\right\|_{2}^{2}+\varepsilon\left\|p^{\varepsilon}\right\|_{2}^{2}$ are non-negative, we then conclude

$$
\begin{aligned}
& \int_{\Omega} \mathbf{S}\left(p^{\varepsilon}, \mathbf{D}\left(\boldsymbol{v}^{\varepsilon}\right)\right) \cdot \mathbf{D}\left(\boldsymbol{v}^{\varepsilon}-\boldsymbol{v}\right) \mathrm{d} x \\
& \leq \int_{\Omega}\left(\boldsymbol{v}^{\varepsilon} \otimes \mathcal{P} \boldsymbol{v}^{\varepsilon}\right) \cdot \nabla\left(\boldsymbol{v}^{\varepsilon}-\boldsymbol{v}\right) \mathrm{d} x-\eta \int_{\Omega}\left|\boldsymbol{v}^{\varepsilon}\right|^{2 r^{\prime}-2} \boldsymbol{v}^{\varepsilon} \cdot\left(\boldsymbol{v}^{\varepsilon}-\boldsymbol{v}\right) \mathrm{d} x+\left\langle\boldsymbol{f}, \boldsymbol{v}^{\varepsilon}-\boldsymbol{v}\right\rangle \\
& =\int_{\Omega}\left(\boldsymbol{v}^{\varepsilon} \otimes \mathcal{P} \boldsymbol{v}^{\varepsilon}\right) \cdot \nabla\left(\boldsymbol{v}^{\varepsilon}-\boldsymbol{v}\right) \mathrm{d} x-\eta\left\|\boldsymbol{v}^{\varepsilon}\right\|_{2 r^{\prime}}^{2 r^{\prime}}+\eta \int_{\Omega}\left|\boldsymbol{v}^{\varepsilon}\right|^{2 r^{\prime}-2} \boldsymbol{v}^{\varepsilon} \cdot \boldsymbol{v} \mathrm{d} x+\left\langle\boldsymbol{f}, \boldsymbol{v}^{\varepsilon}-\boldsymbol{v}\right\rangle .
\end{aligned}
$$

Consequently, we find (after using the weak lower semicontinuity of norms and convergence results (5.1)-(5.2)) that

$$
\limsup _{\varepsilon \rightarrow 0} \int_{\Omega} \mathbf{S}\left(p^{\varepsilon}, \mathbf{D}\left(\boldsymbol{v}^{\varepsilon}\right)\right) \cdot \mathbf{D}\left(\boldsymbol{v}^{\varepsilon}-\boldsymbol{v}\right) \mathrm{d} x \leq-\int_{\Omega}(\boldsymbol{v} \otimes \boldsymbol{v}) \cdot \nabla \boldsymbol{v} \mathrm{d} x=0 .
$$

Hence, in view of (5.13) together with (5.12), the condition (5.10) can be expressed as

$$
\frac{C_{1}}{2} \int_{\Omega} \mathcal{I}^{\mathbf{D}\left(\boldsymbol{v}^{\varepsilon}\right), \mathbf{D}(\boldsymbol{v})} \mathrm{d} x \leq g(\varepsilon)+\frac{\gamma_{0}^{2}}{2 C_{1}}\left\|p_{1}^{\varepsilon}-p_{1}\right\|_{2}^{2}
$$

with $g(\varepsilon) \rightarrow 0$ as $\varepsilon \rightarrow 0$.

In order to handle the term $\left\|p_{1}^{\varepsilon}-p_{1}\right\|_{2}^{2}$, we consider the weak formulation of (3.1) for $\boldsymbol{h}_{1}^{\varepsilon}$ with the special test function $\varphi^{\varepsilon}:=\mathcal{B}\left(p_{1}^{\varepsilon}-p_{1}\right)$. Note that $\int_{\Omega}\left(p_{1}^{\varepsilon}-p_{1}\right) \mathrm{d} x=0$. Since $p_{1}^{\varepsilon} \rightarrow p_{1}$ weakly in $L^{r^{\prime}}(\Omega)$, from the continuity and linearity of the Bogovskil operator we see that

$$
\boldsymbol{\varphi}^{\varepsilon} \rightarrow \mathbf{0} \quad \text { weakly in } W_{0}^{1, r^{\prime}}(\Omega)^{d} .
$$

Thus, testing (3.1) for $\boldsymbol{h}:=\boldsymbol{h}_{1}$ by $\boldsymbol{\varphi}^{\varepsilon}$ we obtain

$$
\begin{aligned}
\left\|p_{1}^{\varepsilon}-p_{1}\right\|_{2}^{2}= & \int_{\Omega} \nabla \boldsymbol{v}_{1}^{\varepsilon} \cdot \nabla \boldsymbol{\varphi}^{\varepsilon} \mathrm{d} x+\int_{\Omega} \mathbf{S}\left(p^{\varepsilon}, \mathbf{D}\left(\boldsymbol{v}^{\varepsilon}\right)\right) \cdot \mathbf{D}\left(\boldsymbol{\varphi}^{\varepsilon}\right) \mathrm{d} x \\
& -\left\langle\boldsymbol{f}, \boldsymbol{\varphi}^{\varepsilon}\right\rangle-\int_{\Omega} p_{1}\left(p_{1}^{\varepsilon}-p_{1}\right) \mathrm{d} x \\
= & \int_{\Omega} \nabla \boldsymbol{v}_{1}^{\varepsilon} \cdot \nabla \boldsymbol{\varphi}^{\varepsilon} \mathrm{d} x+\int_{\Omega} \mathbf{S}\left(p_{1}+p_{2}^{\varepsilon}, \mathbf{D}(\boldsymbol{v})\right) \cdot \mathbf{D}\left(\boldsymbol{\varphi}^{\varepsilon}\right) \mathrm{d} x \\
& +\int_{\Omega}\left(\mathbf{S}\left(p^{\varepsilon}, \mathbf{D}\left(\boldsymbol{v}^{\varepsilon}\right)\right)-\mathbf{S}\left(p_{1}+p_{2}^{\varepsilon}, \mathbf{D}(\boldsymbol{v})\right)\right) \cdot \mathbf{D}\left(\boldsymbol{\varphi}^{\varepsilon}\right) \mathrm{d} x \\
& -\left\langle\boldsymbol{f}, \boldsymbol{\varphi}^{\varepsilon}\right\rangle-\int_{\Omega} p_{1}\left(p_{1}^{\varepsilon}-p_{1}\right) \mathrm{d} x .
\end{aligned}
$$

From (5.15) and from $(5.7)_{2}$ we deduce that

$$
\lim _{\varepsilon \rightarrow 0}\left(\left\langle\boldsymbol{f}, \boldsymbol{\varphi}^{\varepsilon}\right\rangle+\int_{\Omega} p_{1}\left(p_{1}^{\varepsilon}-p_{1}\right) \mathrm{d} x\right)=0 .
$$


Moreover, using the same arguments as in (5.11) and (5.12), we get

$$
\lim _{\varepsilon \rightarrow 0} \int_{\Omega} \mathbf{S}\left(p_{1}+p_{2}^{\varepsilon}, \mathbf{D}(\boldsymbol{v})\right) \cdot \mathbf{D}\left(\boldsymbol{\varphi}^{\varepsilon}\right) \mathrm{d} x=0 .
$$

Since $\nabla \boldsymbol{\varphi}^{\varepsilon} \rightarrow \mathbf{0}$ weakly in $L^{r^{\prime}}(\Omega)^{d \times d}$ and $r^{\prime}>2, \nabla \boldsymbol{\varphi}^{\varepsilon} \rightarrow \mathbf{0}$ weakly in $L^{2}(\Omega)^{d \times d}$ as well. Using $(5.7)_{1},(5.9)$ and the fact that $r^{\prime}>2$, we derive that $\nabla \boldsymbol{v}_{1}^{\varepsilon} \rightarrow \nabla \boldsymbol{v}_{1}$ strongly in $L^{2}(\Omega)^{d \times d}$. Consequently,

$$
\lim _{\varepsilon \rightarrow 0} \int_{\Omega} \nabla \boldsymbol{v}_{1}^{\varepsilon} \cdot \nabla \boldsymbol{\varphi}^{\varepsilon} \mathrm{d} x=0 .
$$

From (5.17), (5.18) and (5.19) we deduce that

$$
\left\|p_{1}^{\varepsilon}-p_{1}\right\|_{2}^{2}=g(\varepsilon)+\int_{\Omega}\left(\mathbf{S}\left(p^{\varepsilon}, \mathbf{D}\left(\boldsymbol{v}^{\varepsilon}\right)\right)-\mathbf{S}\left(p_{1}+p_{2}^{\varepsilon}, \mathbf{D}(\boldsymbol{v})\right)\right) \cdot \mathbf{D}\left(\boldsymbol{\varphi}^{\varepsilon}\right) \mathrm{d} x
$$

where $g(\varepsilon) \rightarrow 0$ for $\varepsilon \rightarrow 0$. After applying the assumptions (2.1) and (2.2) to the integral on the right-hand side, we obtain

$$
\begin{aligned}
& \left\|p_{1}^{\varepsilon}-p_{1}\right\|_{2}^{2} \\
& \leq g(\varepsilon)+\gamma_{0} \int_{\Omega}\left|p_{1}^{\varepsilon}-p_{1}\right|\left|\mathbf{D}\left(\boldsymbol{\varphi}^{\varepsilon}\right)\right| \mathrm{d} x \\
& \quad+C_{2} \int_{\Omega} \int_{0}^{1}\left(1+\left|\mathbf{D}(\boldsymbol{v})+s\left(\mathbf{D}\left(\boldsymbol{v}^{\varepsilon}\right)-\mathbf{D}(\boldsymbol{v})\right)\right|^{2}\right)^{\frac{r-2}{2}}\left|\mathbf{D}\left(\boldsymbol{v}^{\varepsilon}\right)-\mathbf{D}(\boldsymbol{v})\right|\left|\mathbf{D}\left(\boldsymbol{\varphi}^{\varepsilon}\right)\right| \mathrm{d} s \mathrm{~d} x .
\end{aligned}
$$

Next, we use the fact that $r<2$ and by Hölder's inequality we get

$$
\begin{aligned}
\left\|p_{1}^{\varepsilon}-p_{1}\right\|_{2}^{2} \leq & g(\varepsilon)+\gamma_{0}\left\|p_{1}^{\varepsilon}-p_{1}\right\|_{2}\left\|\nabla \boldsymbol{\varphi}^{\varepsilon}\right\|_{2}+C_{2}\left(\int_{\Omega} \mathcal{I}^{\mathbf{D}\left(\boldsymbol{v}^{\varepsilon}\right), \mathbf{D}(\boldsymbol{v})} \mathrm{d} x\right)^{\frac{1}{2}}\left\|\nabla \boldsymbol{\varphi}^{\varepsilon}\right\|_{2} \\
\leq & g(\varepsilon)+\gamma_{0} C_{\operatorname{div}}(\Omega, 2)\left\|p_{1}^{\varepsilon}-p_{1}\right\|_{2}^{2} \\
& +C_{2} C_{\operatorname{div}}(\Omega, 2)\left(\int_{\Omega} \mathcal{I}^{\mathbf{D}\left(\boldsymbol{v}^{\varepsilon}\right), \mathbf{D}(\boldsymbol{v})} \mathrm{d} x\right)^{\frac{1}{2}}\left\|p_{1}^{\varepsilon}-p_{1}\right\|_{2} .
\end{aligned}
$$

Application of Young's inequality gives

$$
\begin{aligned}
\left(1-\gamma_{0} C_{\text {div }}(\Omega, 2)\right)\left\|p_{1}^{\varepsilon}-p_{1}\right\|_{2}^{2} \leq & g(\varepsilon)+\frac{1-\gamma_{0} C_{\text {div }}(\Omega, 2)}{2}\left\|p_{1}^{\varepsilon}-p_{1}\right\|_{2}^{2} \\
& +\frac{C_{2}^{2} C_{\text {div }}^{2}(\Omega, 2)}{2\left(1-\gamma_{0} C_{\text {div }}(\Omega, 2)\right)} \int_{\Omega} \mathcal{I}^{\mathbf{D}\left(\boldsymbol{v}^{\varepsilon}\right), \mathbf{D}(\boldsymbol{v})} \mathrm{d} x .
\end{aligned}
$$

As $1-\gamma_{0} C_{\text {div }}(\Omega, 2)-\frac{1-\gamma_{0} C_{\text {div }}(\Omega, 2)}{2}>0$ due to $(2.6)$, we then conclude

$$
\left\|p_{1}^{\varepsilon}-p_{1}\right\|_{2}^{2} \leq g(\varepsilon)+\frac{C_{2}^{2} C_{\mathrm{div}}^{2}(\Omega, 2)}{\left(1-\gamma_{0} C_{\mathrm{div}}(\Omega, 2)\right)^{2}} \int_{\Omega} \mathcal{I}^{\mathbf{D}\left(\boldsymbol{v}^{\varepsilon}\right), \mathbf{D}(\boldsymbol{v})} \mathrm{d} x .
$$


Coming back to (5.14) and incorporating (5.20), we finally get

$$
\frac{C_{1}}{2} \int_{\Omega} \mathcal{I}^{\mathbf{D}\left(\boldsymbol{v}^{\varepsilon}\right), \mathbf{D}(\boldsymbol{v})} \mathrm{d} x \leq g(\varepsilon)+\frac{\gamma_{0}^{2}}{2 C_{1}} \frac{C_{2}^{2} C_{\mathrm{div}}^{2}(\Omega, 2)}{\left(1-\gamma_{0} C_{\mathrm{div}}(\Omega, 2)\right)^{2}} \int_{\Omega} \mathcal{I}^{\mathbf{D}\left(\boldsymbol{v}^{\varepsilon}\right), \mathbf{D}(\boldsymbol{v})} \mathrm{d} x
$$

with $g(\varepsilon) \rightarrow 0$ for $\varepsilon \rightarrow 0$. As $\frac{C_{1}}{2}-\frac{\gamma_{0}^{2}}{2 C_{1}} \frac{C_{2}^{2} C_{\text {div }}^{2}(\Omega, 2)}{\left(1-\gamma_{0} C_{\text {div }}(\Omega, 2)\right)^{2}}>0$, again thanks to (2.6), we indeed have

$$
\int_{\Omega} \mathcal{I}^{\mathbf{D}\left(\boldsymbol{v}^{\varepsilon}\right), \mathbf{D}(\boldsymbol{v})} \mathrm{d} x \rightarrow 0 \quad \text { for } \varepsilon \rightarrow 0 .
$$

The almost everywhere convergence (at least for a subsequence) of the pressure $p^{\varepsilon}$ in $\Omega$ then follows from (5.8) since (5.21) and (5.20) imply the a.e. convergence of the pressure $p_{1}^{\varepsilon}$ and from $(5.7)_{4}$ also the pressure $p_{2}^{\varepsilon}$ converges a.e. in $\Omega$. Following step by step the same scheme as in [6], one can easily show that (5.21) implies (at least for subsequence)

$$
\mathbf{D}\left(\boldsymbol{v}^{\varepsilon}\right) \rightarrow \mathbf{D}(\boldsymbol{v}) \text { a.e. in } \Omega \text {. }
$$

Using Vitali's theorem, we then conclude that $\overline{\mathbf{S}}=\mathbf{S}(p, \mathbf{D}(\boldsymbol{v}))$.

5.2. Limit $\eta \rightarrow 0$. In this subsection we use the result proven in Subsection 5.1, namely the equation (5.5). Purely from technical reasons, let us first take $\eta:=\frac{1}{n}$. Then we indeed know that there exists a couple $\left(\boldsymbol{v}^{n}, p^{n}\right) \in\left(W_{0, \operatorname{div}}^{1, r}(\Omega)^{d} \cap\right.$ $\left.L^{2 r^{\prime}}(\Omega)^{d}\right) \times L_{0}^{\frac{2 d r}{(d-2) r+d}}(\Omega)$ such that ${ }^{4}$

$$
\begin{aligned}
& \frac{1}{n} \int_{\Omega}\left|\boldsymbol{v}^{n}\right|^{2 r^{\prime}-2} \boldsymbol{v}^{n} \cdot \boldsymbol{\varphi} \mathrm{d} x+\int_{\Omega} \mathbf{S}\left(p^{n}, \mathbf{D}\left(\boldsymbol{v}^{n}\right)\right) \cdot \mathbf{D}(\boldsymbol{\varphi}) \mathrm{d} x-\int_{\Omega} p^{n} \operatorname{div} \boldsymbol{\varphi} \mathrm{d} x \\
& -\int_{\Omega}\left(\boldsymbol{v}^{n} \otimes \boldsymbol{v}^{n}\right) \cdot \nabla \boldsymbol{\varphi} \mathrm{d} x=\langle\boldsymbol{f}, \boldsymbol{\varphi}\rangle \quad \text { for all } \boldsymbol{\varphi} \in W_{0}^{1, \frac{2 d r}{(d+2) r-d}}(\Omega)^{d} .
\end{aligned}
$$

Note that applying the density of smooth functions argument, we also have that (5.22) is valid for all $\boldsymbol{\varphi} \in W_{0}^{1, r}(\Omega)^{d} \cap L^{2 r^{\prime}}(\Omega)^{d}$. Next, taking $\boldsymbol{\varphi}:=\boldsymbol{v}^{n}$ in (5.22) and using the same arguments as in (4.7), we verify

$$
C\left\|\nabla \boldsymbol{v}^{n}\right\|_{r}^{r}+\frac{1}{n}\left\|\boldsymbol{v}^{n}\right\|_{2 r^{\prime}}^{2 r^{\prime}} \leq C<\infty,
$$

and then again from (2.5) with $\lambda=1$ and (5.23) we have that

$$
\left\|\mathbf{S}\left(p^{n}, \mathbf{D}\left(\boldsymbol{v}^{n}\right)\right)\right\|_{r^{\prime}} \leq C<\infty .
$$

In order to obtain an estimate for the pressure $p^{n}$ independent of $n$, we apply the same procedure as for $p^{\varepsilon}$, namely we take the test function $\varphi=\varphi^{n}:=$

\footnotetext{
${ }^{4}$ In formula (5.5) the test functions $\varphi$ are taken from $W_{0}^{1, r}(\Omega)^{d} \cap W^{1, \infty}(\Omega)^{d}$. But once having (5.1), one can easily deduce by using the density of smooth functions that formula (5.5) (and consequently also (5.22)) is valid for all $\varphi \in W_{0}^{1, \frac{2 d r}{(d+2) r-d}}(\Omega)^{d}$.
} 
$\mathcal{B}\left(\left|p^{n}\right|^{s-2} p^{n}-\frac{1}{|\Omega|} \int_{\Omega}\left|p^{n}\right|^{s-2} p^{n} \mathrm{~d} x\right)$ in (5.22) with $s:=\frac{d r}{2(d-r)}$. The function $\varphi^{n}$ then fulfills

$$
\left\|\varphi^{n}\right\|_{1, s^{\prime}} \leq 2 C_{\text {div }}\left(\Omega, s^{\prime}\right)\left\|p^{n}\right\|_{s}^{s-1} .
$$

Note that $\int_{\Omega} p^{n} \mathrm{~d} x=0$. With the help of (2.5), (5.23), (5.25), the embedding $W^{1, s_{1}} \hookrightarrow L^{2 r^{\prime}}(\Omega), s_{1}=\frac{2 d r}{(d+2) r-d}$, and the fact that $s_{1} \leq s^{\prime}$ and $\frac{r+1}{2 r} \leq 1$ we conclude

$$
\begin{aligned}
\left\|p^{n}\right\|_{s}^{s}= & \int_{\Omega} \mathbf{S}\left(p^{n}, \mathbf{D}\left(\boldsymbol{v}^{n}\right)\right) \cdot \mathbf{D}\left(\boldsymbol{\varphi}^{n}\right) \mathrm{d} x-\left\langle\boldsymbol{f}, \boldsymbol{\varphi}^{n}\right\rangle \\
& -\int_{\Omega}\left(\boldsymbol{v}^{n} \otimes \boldsymbol{v}^{n}\right) \cdot \nabla \boldsymbol{\varphi}^{n} \mathrm{~d} x+\frac{1}{n} \int_{\Omega}\left|\boldsymbol{v}^{n}\right|^{2 r^{\prime}-2} \boldsymbol{v}^{n} \cdot \boldsymbol{\varphi}^{n} \mathrm{~d} x \\
\leq & C\left(1+\left\|\mathbf{D}\left(\boldsymbol{v}^{n}\right)\right\|_{r}\right)^{r-1}\left\|\boldsymbol{\varphi}^{n}\right\|_{1, r}+\|\boldsymbol{f}\|_{-1, r^{\prime}}\left\|\boldsymbol{\varphi}^{n}\right\|_{1, r} \\
& +\left\|\boldsymbol{v}^{n} \otimes \boldsymbol{v}^{n}\right\|_{s}\left\|\nabla \boldsymbol{\varphi}^{n}\right\|_{s^{\prime}}+\frac{1}{n}\left\|\boldsymbol{v}^{n}\right\|_{2 r^{\prime}}^{2 r^{\prime}-1}\left\|\boldsymbol{\varphi}^{n}\right\|_{2 r^{\prime}} \\
\leq & C\left\|\boldsymbol{\varphi}^{n}\right\|_{1, s^{\prime}}+C \frac{1}{n}\left(\left\|\boldsymbol{v}^{n}\right\|_{2 r^{\prime}}^{2 r^{\prime}} \frac{r+1}{2 r}\left\|\boldsymbol{\varphi}^{n}\right\|_{1, s_{1}}\right. \\
\leq & C\left\|\boldsymbol{\varphi}^{n}\right\|_{1, s^{\prime}} \\
\leq & C\left\|p^{n}\right\|_{s}^{s-1},
\end{aligned}
$$

which leads to

$$
\left\|p^{n}\right\|_{\frac{d r}{2(d-r)}} \leq C<\infty .
$$

Owing to (5.23), (5.24) and (5.26) we can again find a (not relabeled) subsequence $\left(\boldsymbol{v}^{n}, p^{n}\right)$ and $(\boldsymbol{v}, p) \in W_{0, \operatorname{div}}^{1, r}(\Omega)^{d} \times L_{0}^{\frac{d r}{2(d-r)}}(\Omega)$ such that

$$
\begin{array}{rr}
\boldsymbol{v}^{n} \rightarrow \boldsymbol{v} & \text { weakly in } W_{0, \text { div }}^{1, r}(\Omega)^{d} \\
p^{n} \rightarrow p & \text { weakly in } L_{0}^{\frac{d r}{2(d-r)}}(\Omega) \\
\mathbf{S}\left(p^{n}, \mathbf{D}\left(\boldsymbol{v}^{n}\right)\right) \rightarrow \overline{\mathbf{S}} & \text { weakly in } L^{r^{\prime}}(\Omega)^{d \times d},
\end{array}
$$

and due to the compact embedding

$$
\boldsymbol{v}^{n} \rightarrow \boldsymbol{v} \quad \text { strongly in } L^{q}(\Omega)^{d} \text { for all } q: 1 \leq q<\frac{d r}{d-r} .
$$

Especially, $\boldsymbol{v}^{n} \rightarrow \boldsymbol{v} \quad$ strongly in $L^{2}(\Omega)^{d}$ (as $r>\frac{2 d}{d+2}$ ). Thus, it is easy to pass to the limit in the convective term and also in all linear terms. Moreover, having (5.23), we directly obtain that

$$
\frac{1}{n} \int_{\Omega}\left|\boldsymbol{v}^{n}\right|^{2 r^{\prime}-2} \boldsymbol{v}^{n} \cdot \boldsymbol{\varphi} \mathrm{d} x \rightarrow 0 \quad \forall \boldsymbol{\varphi} \in L^{\infty}(\Omega)^{d} .
$$

Thus, to prove (2.7) it remains to show that $\overline{\mathbf{S}}=\mathbf{S}(p, \mathbf{D}(\boldsymbol{v}))$. Similarly as before, it is enough to know that

$$
p^{n} \rightarrow p \quad \text { a.e. in } \Omega \quad \text { and } \quad \mathbf{D}\left(\boldsymbol{v}^{n}\right) \rightarrow \mathbf{D}(\boldsymbol{v}) \text { a.e. in } \Omega,
$$


at least for a subsequence. Vitali's theorem then completes the whole proof.

In order to show (5.29), we again decompose the pressure $p^{n}$ into two parts and recall the monotone properties of $\mathbf{S}(2.3)$. However, we use this condition in a different way as in the previous section. We apply the so-called Lipschitz approximations of Sobolev functions that (as we are going to see) are essential for the proof.

Consider again two auxiliary Stokes problems (3.1) with the right-hand sides $\boldsymbol{h}_{i}=\boldsymbol{h}_{i}^{n}(i=1,2)$ of the form

$$
\begin{array}{ll}
\boldsymbol{h}_{1}^{n}=\operatorname{div}\left(\mathbf{S}\left(p^{n}, \mathbf{D}\left(\boldsymbol{v}^{n}\right)\right)\right)+\boldsymbol{f} & \in\left(W_{0}^{1, r}(\Omega)\right)^{*} \\
\boldsymbol{h}_{2}^{n}=-\operatorname{div}\left(\boldsymbol{v}^{n} \otimes \boldsymbol{v}^{n}\right)-\frac{1}{n}\left|\boldsymbol{v}^{n}\right|^{2 r^{\prime}-2} \boldsymbol{v}^{n} \in\left(W_{0}^{1, s^{\prime}}(\Omega)\right)^{*}, \quad s^{\prime}=\frac{d r}{(d+2) r-2 d},
\end{array}
$$

and denote $\left(\boldsymbol{v}_{i}^{n}, p_{i}^{n}\right):=\mathcal{S}\left(\boldsymbol{h}_{i}^{n}\right), i=1,2$. Similarly as before, we again have $\boldsymbol{v}_{1}^{n}=-\boldsymbol{v}_{2}^{n}$ and $p^{n}=p_{1}^{n}+p_{2}^{n}$ and we also obtain the following estimates, uniformly w.r.t. $n$ :

$$
\begin{aligned}
&\left\|\nabla \boldsymbol{v}_{1}^{n}\right\|_{r^{\prime}}+\left\|p_{1}^{n}\right\|_{r^{\prime}} \leq C\left\|\boldsymbol{h}_{1}^{n}\right\|_{\left(W_{0}^{1, r}(\Omega)\right)^{*}} \leq C+C\left\|\mathbf{S}\left(p^{n}, \mathbf{D}\left(\boldsymbol{v}^{n}\right)\right)\right\|_{r^{\prime}} \stackrel{(5.24)}{\leq} C \\
&\left\|\nabla \boldsymbol{v}_{2}^{n}\right\|_{s}+\left\|p_{2}^{n}\right\|_{s} \leq C\left\|\boldsymbol{h}_{2}^{n}\right\|_{\left(W_{0}^{1, s^{\prime}}(\Omega)\right)^{*}} \leq C\left\|\boldsymbol{v}^{n}\right\|_{2 s}^{2}+C \frac{1}{n^{\frac{1}{2 r^{\prime}}}}\left(\frac{1}{n}\left\|\boldsymbol{v}^{n}\right\|_{2 r^{\prime}}^{2 r^{\prime}}\right)^{\frac{r+1}{2 r}} \stackrel{(5.23)}{\leq} C .
\end{aligned}
$$

From these inequalities thanks to (5.28) we get

$$
\begin{aligned}
\nabla \boldsymbol{v}_{1}^{n} & \rightarrow \nabla \boldsymbol{v}_{1} & & \text { weakly in } L^{r^{\prime}}(\Omega)^{d \times d} \\
p_{1}^{n} & \rightarrow p_{1} & & \text { weakly in } L^{r^{\prime}}(\Omega) \\
\nabla \boldsymbol{v}_{2}^{n} & \rightarrow \nabla \boldsymbol{v}_{2} & & \text { strongly in } L^{q}(\Omega)^{d \times d} \\
p_{2}^{n} & \rightarrow p_{2} & & \text { strongly in } L^{q}(\Omega),
\end{aligned}
$$

where $q \in\left[1, \frac{d r}{2(d-r)}\right)$. And again from the fact that $\boldsymbol{v}_{1}^{n}=-\boldsymbol{v}_{2}^{n}$ we obtain $\nabla \boldsymbol{v}_{1}^{n} \rightarrow \nabla \boldsymbol{v}_{1}$ strongly in $L^{q}(\Omega)^{d \times d}$.

We want to apply the similar monotone operator argument as in the preceding subsection but now we cannot directly use $\boldsymbol{\varphi}=\boldsymbol{v}^{n}-\boldsymbol{v}$ as a test function in order to treat the term $\int_{\Omega} \mathbf{S}\left(p^{n}, \mathbf{D}\left(\boldsymbol{v}^{n}\right)\right) \cdot \mathbf{D}\left(\boldsymbol{v}^{n}-\boldsymbol{v}\right) \mathrm{d} x$. This is due to the fact that we are interested in the case when $\frac{2 d}{d+2}<r \leq \frac{3 d}{d+2}$ and for this range of $r$ 's the function $\boldsymbol{v}^{n}-\boldsymbol{v}$ is not an admissible test function anymore. The trouble is caused by the convective term since $\int_{\Omega}\left(\boldsymbol{v}^{n} \otimes \boldsymbol{v}^{n}\right) \cdot \nabla\left(\boldsymbol{v}^{n}-\boldsymbol{v}\right) \mathrm{d} x \rightarrow 0$ provided that $r>\frac{3 d}{d+2}$. Nevertheless, we notice that thanks to $(5.27)_{1}$ the functions

$$
\boldsymbol{u}^{n}:=\boldsymbol{v}^{n}-\boldsymbol{v}
$$

satisfy the assumptions of Lemma 3.2 on Lipschitz approximations of functions from $W_{0}^{1, r}(\Omega)^{d}$. Therefore, there exists a sequence $\boldsymbol{u}^{n, j} \in W_{0}^{1, \infty}(\Omega)^{d}$ possessing 
the properties (3.3)-(3.7). Of course,

$$
\Omega=\left\{\boldsymbol{u}^{n}=\boldsymbol{u}^{n, j}\right\} \cup\left\{\boldsymbol{u}^{n} \neq \boldsymbol{u}^{n, j}\right\}=: U_{n, j} \cup\left(\Omega \backslash U_{n, j}\right) .
$$

On returning to $(2.3)$ with $\mathbf{A}:=\mathbf{D}\left(\boldsymbol{v}^{n}\right), \mathbf{B}:=\mathbf{D}(\boldsymbol{v}), p:=p^{n}$ and $q:=p_{1}+p_{2}^{n}$, and integrating it over the set of coincidence $U_{n, j}$, we obtain ( $\chi$ denotes the characteristic function)

$$
\begin{aligned}
\frac{C_{1}}{2} \int_{U_{n, j}} \mathcal{I}^{\mathbf{D}\left(\boldsymbol{v}^{n}\right), \mathbf{D}(\boldsymbol{v})} \mathrm{d} x \leq & \int_{U_{n, j}}\left(\mathbf{S}\left(p^{n}, \mathbf{D}\left(\boldsymbol{v}^{n}\right)\right)-\mathbf{S}\left(p_{1}+p_{2}^{n}, \mathbf{D}(\boldsymbol{v})\right)\right) \cdot \mathbf{D}\left(\boldsymbol{u}^{n, j}\right) \mathrm{d} x \\
& +\frac{\gamma_{0}^{2}}{2 C_{1}}\left\|\left(p_{1}^{n}-p_{1}\right) \chi_{U_{n, j}}\right\|_{2}^{2}
\end{aligned}
$$

Our goal is to show that $\lim \sup _{n \rightarrow \infty} \int_{U_{n, j}} \mathcal{I}^{\mathbf{D}\left(\boldsymbol{v}^{n}\right), \mathbf{D}(\boldsymbol{v})} \mathrm{d} x \leq \varepsilon_{j}$.

Firstly, in the same manner as before we deal with the second integral on the right-hand side. Since Lebesgue's dominated convergence theorem implies that $\mathbf{S}\left(p_{1}+p_{2}^{n}, \mathbf{D}(\boldsymbol{v})\right) \rightarrow \mathbf{S}(p, \mathbf{D}(\boldsymbol{v}))$ strongly in $L^{r^{\prime}}(\Omega)^{d \times d}$, we obtain that $\lim \sup _{n \rightarrow \infty} \int_{\Omega} \mathbf{S}\left(p_{1}+p_{2}^{n}, \mathbf{D}(\boldsymbol{v})\right) \cdot \mathbf{D}\left(\boldsymbol{u}^{n, j}\right) \mathrm{d} x=0$. This implies that

$$
\begin{aligned}
& \limsup _{n \rightarrow \infty} \int_{U_{n, j}} \mathbf{S}\left(p_{1}+p_{2}^{n}, \mathbf{D}(\boldsymbol{v})\right) \cdot \mathbf{D}\left(\boldsymbol{u}^{n, j}\right) \mathrm{d} x \\
& \leq \underset{n \rightarrow \infty}{\limsup }\left|\int_{\Omega \backslash U_{n, j}} \mathbf{S}\left(p_{1}+p_{2}^{n}, \mathbf{D}(\boldsymbol{v})\right) \cdot \mathbf{D}\left(\boldsymbol{u}^{n, j}\right) \mathrm{d} x\right| \\
& \leq C \limsup _{n \rightarrow \infty}\left(\int_{\Omega \backslash U_{n, j}}\left|\mathbf{D}\left(\boldsymbol{u}^{n, j}\right)\right|_{r}^{r} \mathrm{~d} x\right)^{\frac{1}{r}} \\
& \stackrel{(3.7)}{\leq} C \varepsilon_{j}
\end{aligned}
$$

with $\varepsilon_{j}:=C 2^{-\frac{j}{r}}$.

Next, since $\boldsymbol{u}^{n, j}$ is not in general divergence-free on the set of non-coincidence $\Omega \backslash U_{n, j}$, we consider the weak formulation (5.22) with the test function

$$
\boldsymbol{\varphi}=\boldsymbol{\varphi}^{n, j}:=\mathcal{P} \boldsymbol{u}^{n, j}=\boldsymbol{u}^{n, j}-\mathcal{B}\left(\operatorname{div} \boldsymbol{u}^{n, j}\right)=: \boldsymbol{u}^{n, j}-\boldsymbol{\psi}^{n, j}
$$

Note that

$$
\left\|\boldsymbol{\psi}^{n, j}\right\|_{1, r} \leq C_{\operatorname{div}}(\Omega, r)\left\|\operatorname{div} \boldsymbol{u}^{n, j} \chi_{\Omega \backslash U_{n, j}}\right\|_{r} \stackrel{(3.7)}{\leq} C \varepsilon_{j} .
$$

In addition, the properties (3.3)-(3.7) of the sequence $\boldsymbol{u}^{n, j}$ and the continuity and linearity of the Bogovskil operator imply that for $j \in \mathbb{N}$ and $n \rightarrow \infty$

$$
\begin{array}{lll}
\boldsymbol{\psi}^{n, j} \rightarrow \mathbf{0} & \text { strongly in } L^{q}(\Omega)^{d} & \forall q \in(1, \infty) \\
\boldsymbol{\psi}^{n, j} \rightarrow \mathbf{0} & \text { weakly in } W_{0}^{1, q}(\Omega)^{d} & \forall q \in(1, \infty),
\end{array}
$$


and

$$
\begin{aligned}
\limsup _{n \rightarrow \infty}\left\|\boldsymbol{\psi}^{n, j}\right\|_{1, r} & \leq C_{\operatorname{div}}(\Omega, r) \limsup _{n \rightarrow \infty}\left\|\operatorname{div} \boldsymbol{u}^{n, j} \chi_{\Omega \backslash U_{n, j}}\right\|_{r} \\
& \leq C_{\operatorname{div}}(\Omega, r) \limsup _{n \rightarrow \infty}\left\|\nabla \boldsymbol{u}^{n, j} \chi_{\Omega \backslash U_{n, j}}\right\|_{r} \\
& \leq C \varepsilon_{j} .
\end{aligned}
$$

Also note that thanks to (5.33) we also have for $j \in \mathbb{N}$ and $n \rightarrow \infty$ that

$$
\begin{array}{lll}
\boldsymbol{\varphi}^{n, j} \rightarrow \mathbf{0} & \text { strongly in } L^{q}(\Omega)^{d} & \forall q \in(1, \infty) \\
\boldsymbol{\varphi}^{n, j} \rightarrow \mathbf{0} & \text { weakly in } W_{0}^{1, q}(\Omega)^{d} & \forall q \in(1, \infty) .
\end{array}
$$

Now, considering (5.22) with the test function $\varphi=\varphi^{n, j}$, we can write

$$
\begin{aligned}
& \int_{\Omega} \mathbf{S}\left(p^{n}, \mathbf{D}\left(\boldsymbol{v}^{n}\right)\right) \cdot \mathbf{D}\left(\boldsymbol{u}^{n, j}\right) \mathrm{d} x \\
& =\left\langle\boldsymbol{f}, \boldsymbol{\varphi}^{n, j}\right\rangle-\frac{1}{n} \int_{\Omega}\left|\boldsymbol{v}^{n}\right|^{2 r^{\prime}-2} \boldsymbol{v}^{n} \cdot \boldsymbol{\varphi}^{n, j} \mathrm{~d} x \\
& \quad+\int_{\Omega}\left(\boldsymbol{v}^{n} \otimes \boldsymbol{v}^{n}\right) \cdot \nabla \boldsymbol{\varphi}^{n, j} \mathrm{~d} x+\int_{\Omega} \mathbf{S}\left(p^{n}, \mathbf{D}\left(\boldsymbol{v}^{n}\right)\right) \cdot \mathbf{D}\left(\boldsymbol{\psi}^{n, j}\right) \mathrm{d} x \\
& :=I_{n, j}^{1}+I_{n, j}^{2}+I_{n, j}^{3}+I_{n, j}^{4} .
\end{aligned}
$$

Letting $n \rightarrow \infty$ and taking (5.23), (5.28) and (5.35) into account, we see that $\lim _{n \rightarrow \infty}\left(I_{n, j}^{1}+I_{n, j}^{2}+I_{n, j}^{3}\right)=0$. On the other hand, on using Hölder's inequality, (5.24) and (5.34), we get

$$
\left|I_{n, j}^{4}\right| \leq\left\|\mathbf{S}\left(p^{n}, \mathbf{D}\left(\boldsymbol{v}^{n}\right)\right)\right\|_{r^{\prime}}\left\|\mathbf{D}\left(\boldsymbol{\psi}^{n, j}\right)\right\|_{r} \stackrel{(5.34)}{\leq} C \frac{\gamma_{n}}{\theta_{n}} \mu_{j+1}+C \varepsilon_{j}=g(n)+C \varepsilon_{j},
$$

where for $j \in \mathbb{N}$ fixed the function $g(n) \rightarrow 0$ for $n \rightarrow \infty$. Using everything together with (5.36), we get that $\limsup _{n \rightarrow \infty} \int_{\Omega} \mathbf{S}\left(p^{n}, \mathbf{D}\left(\boldsymbol{v}^{n}\right)\right) \cdot \mathbf{D}\left(\boldsymbol{u}^{n, j}\right) \mathrm{d} x \leq C \varepsilon_{j}$. Moreover, Hölder's inequality, (5.24) and (3.7) yield that also

$$
\limsup _{n \rightarrow \infty}\left|\int_{\Omega \backslash U_{n, j}} \mathbf{S}\left(p^{n}, \mathbf{D}\left(\boldsymbol{v}^{n}\right)\right) \cdot \mathbf{D}\left(\boldsymbol{u}^{n, j}\right) \mathrm{d} x\right| \leq C \varepsilon_{j},
$$

and therefore

$$
\limsup _{n \rightarrow \infty} \int_{U_{n, j}} \mathbf{S}\left(p^{n}, \mathbf{D}\left(\boldsymbol{v}^{n}\right)\right) \cdot \mathbf{D}\left(\boldsymbol{u}^{n, j}\right) \mathrm{d} x \leq C \varepsilon_{j} .
$$

Coming back to the inequality (5.31), using (5.32) and (5.37) we then conclude

$$
\begin{aligned}
\frac{C_{1}}{2} \int_{U_{n, j}} \mathcal{I}^{\mathbf{D}\left(\boldsymbol{v}^{n}\right), \mathbf{D}(\boldsymbol{v})} \mathrm{d} x & \leq g(n)+C \varepsilon_{j}+\frac{\gamma_{0}^{2}}{2 C_{1}}\left\|\left(p_{1}^{n}-p_{1}\right) \chi_{U_{n, j}}\right\|_{2}^{2} \\
& \leq g(n)+C \varepsilon_{j}+\frac{\gamma_{0}^{2}}{2 C_{1}}\left\|p_{1}^{n}-p_{1}\right\|_{2}^{2}
\end{aligned}
$$


Clearly, we would also like to have the estimate $\left\|p_{1}^{n}-p_{1}\right\|_{2}^{2} \leq g(n)+C \varepsilon_{j}$. For this purpose, we first look at the set $\Omega \backslash U_{n, j}=\left\{\boldsymbol{u}^{n} \neq \boldsymbol{u}^{n, j}\right\}$ and show that its Lebesgue measure is sufficiently small. We recall the Theorem 3.2 and since $\lambda_{n, j} \geq 1$, according to (3.2), we observe from (3.7) that

$$
\begin{aligned}
\left|\Omega \backslash U_{n, j}\right|=\left\|\chi_{\Omega \backslash U_{n, j}}\right\|_{1} & \leq c \lambda_{n, j}^{-1}\left\|\lambda_{n, j} \chi_{\Omega \backslash U_{n, j}}\right\|_{r} \\
& \leq c\left\|\lambda_{n, j} \chi_{\Omega \backslash U_{n, j}}\right\|_{r} \\
& \leq g(n)+c \varepsilon_{j} .
\end{aligned}
$$

Next, we consider the weak formulation of (3.1) for $\boldsymbol{h}_{1}^{n}$ with the test function $\varphi^{n}:=\mathcal{B}\left(p_{1}^{n}-p_{1}\right)$. Notice that $\int_{\Omega}\left(p_{1}^{n}-p_{1}\right) \mathrm{d} x=0$. From the properties of the Bogovskil operator and from $(5.30)_{2}$ we have $\boldsymbol{\varphi}^{n} \rightarrow \mathbf{0}$ weakly in $W^{1, r^{\prime}}(\Omega)^{d}$. Using the same arguments as for (5.17), (5.18) and (5.19), we obtain

$$
\left\|p_{1}^{n}-p_{1}\right\|_{2}^{2}=g(n)+\int_{\Omega}\left(\mathbf{S}\left(p^{n}, \mathbf{D}\left(\boldsymbol{v}^{n}\right)\right)-\mathbf{S}\left(p_{1}+p_{2}^{n}, \mathbf{D}(\boldsymbol{v})\right)\right) \cdot \mathbf{D}\left(\boldsymbol{\varphi}^{n}\right) \mathrm{d} x,
$$

where $g(n) \rightarrow 0$ for $n \rightarrow \infty$. From the application of (2.1) and (2.2) to the integral on the right-hand side and Hölder's inequality we get

$$
\left\|p_{1}^{n}-p_{1}\right\|_{2}^{2} \leq g(n)+\gamma_{0} C_{\text {div }}(\Omega, 2)\left\|p_{1}^{n}-p_{1}\right\|_{2}^{2}+C_{2} \int_{\Omega} \mathcal{J} \mathrm{d} x
$$

where $\mathcal{J}:=\int_{0}^{1}\left(1+\left|\mathbf{D}(\boldsymbol{v})+s\left(\mathbf{D}\left(\boldsymbol{v}^{n}-\boldsymbol{v}\right)\right)\right|^{2}\right)^{\frac{r-2}{2}}\left|\mathbf{D}\left(\boldsymbol{v}^{n}-\boldsymbol{v}\right)\right|\left|\mathbf{D}\left(\boldsymbol{\varphi}^{n}\right)\right| \mathrm{d} s$. We split the integral in (5.40) as $\int_{\Omega} \mathcal{J} \mathrm{d} x=\int_{U_{n, j}} \mathcal{J} \mathrm{d} x+\int_{\Omega \backslash U_{n, j}} \mathcal{J} \mathrm{d} x$. Using Hölder's inequality, (2.1), (2.2) and Young's inequality, we can write

$$
\begin{aligned}
\int_{U_{n, j}} \mathcal{J} \mathrm{d} x & \leq C_{\mathrm{div}}(\Omega, 2)\left(\int_{U_{n, j}} \mathcal{I}^{\mathbf{D}\left(\boldsymbol{v}^{n}\right), \mathbf{D}(\boldsymbol{v})} \mathrm{d} x\right)^{\frac{1}{2}}\left\|p_{1}^{n}-p_{1}\right\|_{2} \\
& \leq \frac{C_{\mathrm{div}}^{2}(\Omega, 2) C_{2}}{2\left(1-\gamma_{0} C_{\mathrm{div}}(\Omega, 2)\right)} \int_{U_{n, j}} \mathcal{I}^{\mathbf{D}\left(\boldsymbol{v}^{n}\right), \mathbf{D}(\boldsymbol{v})} \mathrm{d} x+\frac{1-\gamma_{0} C_{\mathrm{div}}(\Omega, 2)}{2 C_{2}}\left\|p_{1}^{n}-p_{1}\right\|_{2}^{2}
\end{aligned}
$$

Applying Hölder's inequality again, using (5.23), (5.39) and the fact that $r<2$ we deduce that

$$
\begin{aligned}
\int_{\Omega \backslash U_{n, j}} \mathcal{J} \mathrm{d} x & \leq\left(\int_{\Omega \backslash U_{n, j}} \mathcal{I}^{\mathbf{D}\left(\boldsymbol{v}^{n}\right), \mathbf{D}(\boldsymbol{v})} \mathrm{d} x\right)^{\frac{1}{2}}\left(\int_{\Omega \backslash U_{n, j}}\left|\nabla \boldsymbol{\varphi}^{n}\right|^{r^{\prime}} \mathrm{d} x\right)^{\frac{1}{r^{\prime}}}\left|\Omega \backslash U_{n, j}\right|^{\frac{r^{\prime}-2}{2 r^{\prime}}} \\
& \leq C\left|\Omega \backslash U_{n, j}\right|^{\frac{r^{\prime}-2}{2 r^{\prime}}} \\
& \leq g(n)+C\left(\varepsilon_{j}\right)^{\frac{r^{\prime}-2}{2 r^{\prime}}} .
\end{aligned}
$$

Putting this together with (5.40) we see that

$$
\left\|p_{1}^{n}-p_{1}\right\|_{2}^{2} \leq g(n)+C\left(\varepsilon_{j}\right)^{\frac{r^{\prime}-2}{2 r^{\prime}}}+\frac{C_{\mathrm{div}}^{2}(\Omega, 2) C_{2}^{2}}{\left(1-\gamma_{0} C_{\mathrm{div}}(\Omega, 2)\right)^{2}} \int_{U_{n, j}} \mathcal{I}^{\mathbf{D}\left(\boldsymbol{v}^{n}\right), \mathbf{D}(\boldsymbol{v})} \mathrm{d} x
$$


Furthermore, as $r^{\prime}>2$ and $\frac{C_{1}}{2}-\frac{\gamma_{0}^{2}}{2 C_{1}} \frac{C_{\mathrm{div}}^{2}(\Omega, 2) C_{2}^{2}}{\left(1-\gamma_{0} C_{\mathrm{div}}(\Omega, 2)\right)^{2}}>0$, again due to $(2.6)$, from (5.38) and (5.41) we indeed have

$$
\limsup _{n \rightarrow \infty} \int_{U_{n, j}} \mathcal{I}^{\mathbf{D}\left(\boldsymbol{v}^{n}\right), \mathbf{D}(\boldsymbol{v})} \mathrm{d} x \leq \varepsilon_{j}
$$

The almost everywhere convergence (at least for a subsequence) of the pressure $p^{n}$ in $\Omega$ then follows from $(5.41),(5.42)$ and $(5.30)_{4}$.

Finally, considering (5.39) and since $\left\|\nabla \boldsymbol{v}^{n}\right\|_{r} \leq C,\|\nabla \boldsymbol{v}\|_{r} \leq C$ and $r<2$, we obtain (again following step by step the same procedure as in [6])

$$
\begin{aligned}
& \int_{\Omega}\left|\mathbf{D}\left(\boldsymbol{v}^{n}-\boldsymbol{v}\right)\right| \mathrm{d} x \\
&=\int_{U_{n, j}}\left|\mathbf{D}\left(\boldsymbol{v}^{n}-\boldsymbol{v}\right)\right| \mathrm{d} x+\int_{\Omega \backslash U_{n, j}}\left|\mathbf{D}\left(\boldsymbol{v}^{n}-\boldsymbol{v}\right)\right| \mathrm{d} x \\
& \leq \int_{U_{n, j}}\left(\mathcal{I}^{\mathbf{D}\left(\boldsymbol{v}^{n}\right), \mathbf{D}(\boldsymbol{v})}\right)^{\frac{1}{2}}\left(\int_{0}^{1}\left(1+\left|\mathbf{D}(\boldsymbol{v})+s\left(\mathbf{D}\left(\boldsymbol{v}^{n}-\boldsymbol{v}\right)\right)\right|^{2}\right)^{\frac{r-2}{2}} \mathrm{~d} s\right)^{-\frac{1}{2}} \mathrm{~d} x \\
& \quad+\int_{\Omega \backslash U_{n, j}}\left(\left|\mathbf{D}\left(\boldsymbol{v}^{n}\right)\right|+|\mathbf{D}(\boldsymbol{v})|\right) \mathrm{d} x \\
& \leq C \int_{U_{n, j}}\left(\mathcal{I}^{\mathbf{D}\left(\boldsymbol{v}^{n}\right), \mathbf{D}(\boldsymbol{v})}\right)^{\frac{1}{2}}\left(1+\left|\mathbf{D}\left(\boldsymbol{v}^{n}\right)\right|+|\mathbf{D}(\boldsymbol{v})|\right)^{\frac{2-r}{2}} \mathrm{~d} x \\
& \quad+\left(\left\|\nabla \boldsymbol{v}^{n}\right\|_{r}+\|\nabla \boldsymbol{v}\|_{r}\right)\left|\Omega \backslash U_{n, j}\right|^{\frac{1}{r^{\prime}}} \\
& \leq C\left(\int_{U_{n, j}} \mathcal{I}^{\mathbf{D}\left(\boldsymbol{v}^{n}\right), \mathbf{D}(\boldsymbol{v})} \mathrm{d} x\right)^{\frac{1}{2}}\left(1+\left\|\nabla \boldsymbol{v}^{n}\right\|_{r}+\|\nabla \boldsymbol{v}\|_{r}\right)^{\frac{2-r}{2}}|\Omega|^{\frac{1}{r^{\prime}}}+g(n)+C\left(\varepsilon_{j}\right)^{\frac{1}{r^{\prime}}} \\
& \leq C\left(\int_{U_{n, j}} \mathcal{I}^{\mathbf{D}\left(\boldsymbol{v}^{n}\right), \mathbf{D}(\boldsymbol{v})} \mathrm{d} x\right)^{\frac{1}{2}}+g(n)+C\left(\varepsilon_{j}\right)^{\frac{1}{r^{\prime}}} .
\end{aligned}
$$

Thus, letting $n \rightarrow \infty$ and then $\varepsilon_{j} \rightarrow 0$ (i.e., $j \rightarrow \infty$ ), we obtain (at least for a subsequence) $\mathbf{D}\left(\boldsymbol{v}^{n}\right) \rightarrow \mathbf{D}(\boldsymbol{v})$ a.e. in $\Omega$. Hence, (5.29) is proven and the proof of Theorem 2.1 is complete.

Acknowledgement. This article is based on the diploma thesis of V. Fišerová that was defended at the Faculty of Mathematics and Physics of the Charles University in Prague. V. Fišerová's research was also partly supported by the Czech Science Foundation, the project GAČR 201/06/0352. M. Bulíček's research is supported by the Jindřich Nečas Center for Mathematical Modeling, the project LC06052 financed by MŠMT.

The authors are also thankful to Josef Málek for his comments to this work. 


\section{References}

[1] Acerbi, E. and Fusco, N., Semicontinuity problems in the calculus of variations. Arch. Rational Mech. Anal. 86 (1984), 125 - 145.

[2] Amrouche, Ch. and Girault, V., Decomposition of vector spaces and application to the Stokes problem in arbitrary dimension. Czechoslovak Math. J. 44 (1994), $109-140$.

[3] Boccardo, L. and Murat, F., Almost everywhere convergence of the gradients of solutions to elliptic and parabolic equations. Nonlinear Anal. 19 (1992)(6), $581-597$.

[4] Bogovskiü, M. E., Solutions of some problems of vector analysis, associated with the operators div and grad (in Russian). In: Trudy Sem. S. L. Soboleva 2. Novosibirsk: Akad. Nauk SSSR Sibirsk. Otdel. 1980, pp. 5 - 40.

[5] Bridgman, P. W., The Physics of High Pressure. New York: MacMillan 1931.

[6] Bulíček, M., Málek, J. and Rajagopal, K. R., Navier's slip and evolutionary Navier-Stokes-like systems with pressure and shear-rate dependent viscosity. Indiana Univ. Math. J. 56 (2007), $51-85$.

[7] Bulíček, M., Málek, J. and Rajagopal, K. R., Mathematical analysis of unsteady flows of fluids with pressure, shear-rate and temperature dependent material moduli, that slip at solid boundaries. To appear in: SIAM J. Math. Anal. (2009).

[8] Diening, L., Málek, J. and Steinhauer, M., On Lipschitz truncations of Sobolev functions (with variable exponent) and their selected applications. ESAIM: Control Optim. Calc. Var. 14 (2008)(2), $211-232$.

[9] Franta, M., Málek, J. and Rajagopal, K. R., On steady flows of fluids with pressure- and shear-dependent viscosities. Proc. R. Soc. Lond. Ser. A Math. Phys. Eng. Sci. 461 (2005)(2055), $651-670$.

[10] Frehse, J., Málek, J. and Steinhauer, M., An existence result for fluids with shear dependent viscosity - steady flows. Nonlin. Analysis TMA 30 (1997), $3041-3049$.

[11] Frehse, J., Málek, J. and Steinhauer, M., On analysis of steady flows of fluids with shear-dependent viscosity based on the Lipschitz truncation method. SIAM J. Math. Anal. 34 (2003)(5), $1064-1083$.

[12] Gazzola, F., A note on the evolution of Navier-Stokes equations with a pressure-dependent viscosity. Z. Angew. Math. Phys. 48 (1997)(5), $760-773$.

[13] Gazzola, F. and Secchi, P., Some results about stationary Navier-Stokes equations with a pressure-dependent viscosity. In: Navier-Stokes Equations: Theory and Numerical Methods (Varenna 1997; ed.: R. Salvi). Pitman Res. Notes Math. Ser. 388. Harlow: Longman 1998, pp. $31-37$.

[14] Hron, J., Málek, J. and Rajagopal, K. R., Simple flows of fluids with pressure dependent viscosities. Proc. R. Soc. Lond. Ser. A Math. Phys. Eng. Sci. 477 (2001), $277-302$. 
[15] Hron, J., Málek, J., Nečas, J. and Rajagopal, K. R., Numerical simulations and global existence of solutions of two-dimensional flows of fluids with pressureand shear-dependent viscosities. Math. Comput. Simulation 61 (2003), 297 - 315.

[16] Ladyženskaja, O. A., New equations for the description of the motions of viscous incompressible fluids, and global solvability for their boundary value problems (in Russian). Trudy Steklov's Math. Institute 102 (1967), 85 - 104.

[17] Ladyženskaja, O. A., Modifications of the Navier-Stokes equations for large gradients of the velocities (in Russian). Zap. Naučn. Sem. Leningrad. Otdel. Mat. Inst. Steklov. (LOMI) 7 (1968), 126 - 154.

[18] Ladyzhenskaya, O. A., The Mathematical Theory of Viscous Incompressible Flow. New York: Gordon \& Breach 1969.

[19] Lanzendörfer, M., On steady inner flows of an incompressible fluid with the viscosity depending on the pressure and the shear rate. Nonlinear Anal. Real World Appl. 10 (2009)(2), 1943 - 1954.

[20] Lions, J.-L., Quelques Méthodes de Résolution des Problèmes aux Limites non Linéaires (in French). Paris: Dunod, Gauthier-Villars 1969.

[21] Málek, J. and Rajagopal, K. R., Mathematical issues concerning the NavierStokes equations and some of its generalizations. In: Evolutionary Equations II. Handb. Diff. Equ. Amsterdam: Elsevier/North-Holland 2005, pp. 371 - 459.

[22] Málek, J. and Rajagopal, K. R., Mathematical properties of the solutions to the equations governing the flow of fluids with pressure and shear rate dependent viscosities. In: Mathematical Theory in Fluid Dynamics IV. Amsterdam: Elsevier/North-Holland 2007, pp. 407 - 444.

[23] Málek, J., Nečas, J., Rokyta, M. and Růžička, M., Weak and Measure-Valued Solutions to Evolutionary PDEs. London: Chapman \& Hall 1996.

[24] Málek, J., Nečas, J. and Rajagopal, K. R., Global analysis of the flows of fluids with pressure-dependent viscosities. Arch. Ration. Mech. Anal. 165 (2002)(3), $243-269$.

[25] Novotný, A. and Straškraba, I., Introduction to the Mathematical Theory of Compressible Flow. Oxford Lecture Ser. Math. Appl. 27. Oxford: Oxford Univ. Press 2004.

[26] Rajagopal, K. R., On implicit constitutive theories. Appl. Math. 48 (2003)(4), $279-319$.

[27] Rajagopal, K. R. and Srinivasa, A. R., On thermomechanical restrictions of continua. Proc. R. Soc. Lond. Ser. A Math. Phys. Eng. Sci. 460 (2004)(2042), $631-651$.

[28] Renardy, M., Some remarks on the Navier-Stokes equations with a pressuredependent viscosity. Comm. Part. Diff. Equ. 11 (1986)(7), $779-793$.

[29] Růžička, M., A note on steady flow of fluids with shear dependent viscosity. Nonlin. Anal. 30 (1997), 3029 - 3039.

Received November 6, 2007 